\title{
BLOCK-SPARSE RECOVERY OF SEMIDEFINITE SYSTEMS AND GENERALIZED NULL SPACE CONDITIONS
}

\author{
JANIN HEUER, FREDERIC MATTER, MARC E. PFETSCH, AND THORSTEN THEOBALD
}

\begin{abstract}
This article considers the recovery of low-rank matrices via a convex nuclearnorm minimization problem and presents two null space properties (NSP) which characterize uniform recovery for the case of block-diagonal matrices and block-diagonal positive semidefinite matrices. These null-space conditions turn out to be special cases of a new general setup, which allows to derive the mentioned NSPs and well-known NSPs from the literature. We discuss the relative strength of these conditions and also present a deterministic class of matrices that satisfies the block-diagonal semidefinite NSP.
\end{abstract}

\section{INTRODUCTION}

The motivating example for this article is the recovery of special solutions of semidefinite systems. Let $A: \mathcal{S}^{n} \rightarrow \mathbb{R}^{m}$ be a linear operator from the real symmetric matrices $\mathcal{S}^{n}$ to $\mathbb{R}^{m}$. The goal is to recover an unknown matrix $X^{(0)}$ from its measurements $A\left(X^{(0)}\right)$ only, that is, we are searching for $X \in \mathcal{S}^{n}$ with $A(X)=A\left(X^{(0)}\right)$. If $A$ is underdetermined, then it is unlikely that $X=X^{(0)}$ is recovered. In order to increase chances of recovery, i.e., $X^{(0)}$ being the unique optimal solution of the corresponding recovery problem, additional information on $X^{(0)}$ can be used, in particular, that it has low rank. In this case, Fazel [17] suggested to solve the optimization problem

$$
\min \left\{\operatorname{rank}(X): A(X)=A\left(X^{(0)}\right), X \in \mathcal{S}^{n}\right\} .
$$

Since the rank is a nonconvex function, (1.1) is hard to solve in practice. Instead, one usually applies a convex relaxation by replacing $\operatorname{rank}(X)$ by the nuclear norm $\|X\|_{*}$, which is defined as $\|X\|_{*}=\sum_{i=1}^{n} \sigma_{i}(X)$, where $\sigma_{i}(X)$ are the singular values of $X$ for $i \in[n]:=\{1, \ldots, n\}$. The corresponding convex optimization problem reads

$$
\min \left\{\|X\|_{*}: A(X)=A\left(X^{(0)}\right), X \in \mathcal{S}^{n}\right\},
$$

see Recht et al. [39] and the references therein.

To further increase the chances of recovery, additional information might be used, e.g., that $X^{(0)}$ is positive semidefinite or that it has a block-diagonal structure; the corresponding optimization problems can easily be formulated.

In fact, the mentioned settings incorporate several important special cases that have been discussed in the compressed sensing literature. For instance, the classical problem of

Date: June 29, 2020.

2010 Mathematics Subject Classification. 94A12, 90C22.

Keywords. Semidefinite system, Null space condition, Block-sparse recovery, Compressed sensing.

The second and the third author acknowledge support by the EXPRESS II project within the DFG priority program CoSIP (DFG-SPP 1798). 
recovering of a sparse vector $x$ from measurements $A(x)$ can be obtained as a special case, since every vector can be interpreted as a diagonal matrix. Since the rank of a matrix $X$ is given by the number of nonzero singular values of $X$, i.e., the $\ell_{0}$-"norm" of the vector of singular values of $X$, a low-rank matrix can be considered as a natural generalization of a sparse vector. Moreover, the nuclear norm can be seen as a generalization of the $\ell_{1}$-norm, which has been used for a convex relaxation in this case, see Chen et al. [6]. We refer to the book by Foucart and Rauhut [18] for an introduction to compressed sensing.

An important problem is to characterize when the convex relaxation (1.2) yields $X^{(0)}$ as a unique solution for any $X^{(0)}$ up to a given sparsity level. Such uniform recovery can be characterized by a so-called null space property (NSP). In the classical case, the corresponding NSP can be found in Gribonval and Nielsen [22] and in [18, Theorem 4.4]. If the vectors have to be nonnegative, the respective NSP appears in Khajehnejad et al. [28] and in Zhang [47]. For the case of arbitrary matrices or positive semidefinite (psd) matrices, corresponding NSPs can be found in Kong et al. [29], Oymak and Hassibi [38], or in [18, Theorem 4.40]. A very general setting for NSPs that subsumes most of the existing NSPs has been introduced by Juditsky et al. [24].

To the best of the authors' knowledge, NSPs for the block-sparse semidefinite case have not been considered in any of the existing literature (see Section 3.1 for a formal definition of block-sparsity). Indeed, one contribution of this article is the introduction of the corresponding NSP. It turned out that one can (with a little work) adapt the proofs of the existing NSPs in the classical cases. A closer investigation led us to the conclusion that all of these conditions can, in fact, be presented and their validity proved in a very general setting, which we describe in this article. Our setting is similar to the one of Juditsky et al. [24], but deviates in some key points in order to also cover nonnegative and positive semidefinite recovery.

Besides the apparent applications in compressed sensing, positive semidefinite systems with a block-diagonal form (as formally defined in Definition 3.2) appear in various other areas. Consider a standard semidefinite problem (SDP)

$$
\min \left\{A_{0} \bullet X: A_{p} \bullet X=b_{p}, p \in\{1, \ldots, m\}, X \succeq 0\right\},
$$

with $A_{0}, \ldots, A_{m} \in \mathcal{S}^{n}, b \in \mathbb{R}^{m}$ and $U \bullet V:=\sum_{i, j=1}^{n} U_{i j} V_{i j}$. To improve solving times for such problems, sparsity in the matrices $A_{p}$ should be exploited. Indeed, it is possible to introduce a block-diagonal form on the matrix $X$, which corresponds to the positions of the nonzero entries in the $A_{p}$ matrices. After a minor reformulation, one ends up with an SDP of the form (1.3), where the matrices are in block-diagonal form. Since no sparsityrelated term is added to the objective function, the optimal solution remains unchanged. For more information on sparsity in SDPs, see, e.g., [19, 35, 46].

Moreover, block-diagonal systems appear when considering structured infeasibility in SDPs as a generalization of the well understood structure of infeasible linear inequality systems, see, e.g., the book [7]. Analogously to the linear case, an irreducible infeasible subsystem (IIS) of a semidefinite system can be defined, that is, an infeasible subsystem such that every proper subsystem is feasible. This can be done using block-diagonal systems, so that an IIS is given by an inclusion-minimal set of infeasible block-diagonal subsystems. In the linear case, it is possible to fully characterize IISs by a theorem of 
Gleeson and Ryan [21]. For semidefinite systems however, it turns out that this is no longer true and subsystems with minimal block-support, i.e., block-sparse subsystems need to be computed in order to find an IIS, see [27] for more details.

Main contributions. 1. We introduce a new generalized setting for recovery problems, which also allows to model nonnegative and positive semidefinite constraints. Our main result is a pair of null space conditions for the exact characterization of the uniform recovery of sufficiently sparse signals from their measurements. See Theorem 2.7, which both provides a comprehensive mathematical answer on the influence of nonnegative and semidefinite constraints and offers a versatile tool set for a wide spectrum of reconstruction scenarios.

2. Building upon our new NSP-framework, we establish an NSP for uniform recovery of positive semidefinite block-diagonal matrices, see Theorem 3.4.

3. We analyze the relative strength of the new null space conditions. To this end, we provide a detailed classification of the most prominent subclasses of NSPs within the general framework, with a particular focus on the block-semidefinite case. This includes an NSP for the nonnegative block case (which had also been open so far, see Section 4.1). In particular, we reveal the additional power of a nonnegative block setting for vectors and a positive semidefinite block setting for matrices, respectively. To achieve this, we construct an infinite family of instances such that the nonnegative block NSP captures cases which are neither captured by the unrestricted block NSP nor by the nonnegative linear NSP, see Theorem 4.5.

The paper is structured as follows. In Section 2, we introduce to the setting of the paper and establish the relevant generalized NSPs. In Section 3, we deal with the block-diagonal semidefinite case. Section 4 then provides the interrelations between the various NSPs. Section 5 concludes the paper with some open questions.

Notation. Throughout the paper, we use the following notation. The set of symmetric real $n \times n$ matrices is denoted by $\mathcal{S}^{n}$ and the set of positive semidefinite real $n \times n$ matrices by $\mathcal{S}_{+}^{n}$. Positive semidefiniteness of a matrix $X$ is shortly written as $X \succeq 0$. The inner

matrix product of $A, B \in \mathcal{S}^{n}$ is defined as $A \bullet B:=\operatorname{tr}\left(A^{\top} B\right)=\sum_{i, j=1}^{n} A_{i j} B_{i j}$, where $\operatorname{tr}(\cdot)$ is the trace.

On the space $\mathbb{R}^{n}$, let $\|x\|_{q}:=\left(\sum_{i=1}^{n}\left|x_{i}\right|^{q}\right)^{1 / q}$ denote the $\ell_{q}$-norm for some $q \geq 1$, and define $\|\cdot\|_{0}:=\left|\left\{i \in[n]: x_{i} \neq 0\right\}\right|$ as the $\ell_{0}$-"norm".

\section{General sparsity Structures}

As in the framework of Juditsky et al. [24], we use a linear sensing map $A: \mathcal{X} \rightarrow \mathbb{R}^{m}$ to observe signals $x \in \mathcal{X}$ and a linear representation map $B: \mathcal{X} \rightarrow \mathcal{E}$ for mapping a signal to an appropriate representation, where $\mathcal{X}$ and $\mathcal{E}$ are Euclidean spaces.

By choosing $\mathcal{X}=\mathcal{E}$ and $B$ to be the identity, this covers the classical setting of sparse recovery, where $x$ is sparse in its "natural" representation. However, the framework also covers the setting where the signal $x$ is only sparse in a suitable representation system, with $B$ being an appropriate transformation; this is called the "analysis setting" in the compressed 
sensing world; for an overview see, e.g., [4, 13, 25, 36]. Examples for transformations include the discrete Fourier transform, different wavelet transforms [3, 23, 32, 41, 42] or a finite difference operator in total variation minimization $[2,5,37]$.

We introduce a set $\mathcal{C}$ capturing further constraints emerging from additional information like nonnegativity, and its image $\mathcal{D}$ under the map $B$. Under natural assumptions, we formulate a null space property for the uniform recovery for the set $\mathcal{C}$ in Section 2.1.

If $\mathcal{C}=\mathcal{X}$, then our framework reduces to the framework of [24], and our statements become the statements concerning noise-free recovery in [24], see Remark 2.8 below.

Remark 2.1. Throughout the paper, we consider real vector spaces $\mathcal{X}$ and $\mathcal{E}$, since this is the more natural setting when considering nonnegative vectors. However, at least for unrestricted (block-)vectors or (block-diagonal)matrices, the null space properties in the subsequent Section 2.1 also carry over without changes to the situation where the spaces $\mathcal{X}$ and $\mathcal{E}$ are complex spaces.

\subsection{A GENERALIZED FRAMEWORK FOR SPARSE RECOVERY UNDER SIDE CONSTRAINTS}

Let $\mathcal{X}, \mathcal{E}$ be finite-dimensional Euclidean spaces and consider an arbitrary set $\mathcal{C} \subseteq \mathcal{X}$ with $0 \in \mathcal{C}$. Let $A: \mathcal{X} \rightarrow \mathbb{R}^{m}$ be a linear sensing map and $B: \mathcal{X} \rightarrow \mathcal{E}$ be a linear representation map. Denote by $\mathcal{D}:=\{B(x): x \in \mathcal{C}\} \subseteq \mathcal{E}$ the image of $\mathcal{C}$ under $B$. Consider a norm $\|\cdot\|$ on $\mathcal{E}$, a set $\mathcal{P}$ of matrices representing linear maps on $\mathcal{E}$ and a map $\nu: \mathcal{P} \rightarrow \mathbb{R}_{+}$. Each map $P \in \mathcal{P}$ is assigned a nonnegative real weight $\nu(P)$ and a linear map $\bar{P}: \mathcal{E} \rightarrow \mathcal{E}$. Note that in many examples, such as the ones in Example 2.3 below, $\nu(P)$ will be integer-valued, but it is not necessary to assume this.

Remark 2.2. In this section, unless otherwise stated, we denote the image $F(x)$ of $x$ under a linear operator $F$ as $F x$.

For some real nonnegative $s$, an element $y \in \mathcal{E}$ is called $s$-sparse if there exists a linear map $P \in \mathcal{P}$ with $\nu(P) \leq s$ and $P y=y$. Accordingly, an element $x \in \mathcal{X}$ is called $s$-sparse, if its representation $B x \in \mathcal{E}$ is $s$-sparse. Define the set $\mathcal{P}_{s}:=\{P \in \mathcal{P}: \nu(P) \leq s\}$ of linear maps that can allow $s$-sparse elements.

For a given right-hand side $b \in \mathbb{R}^{m}$, the generalized recovery problem now reads

$$
\min \{\|B x\|: A x=b, x \in \mathcal{C}\} .
$$

If $\mathcal{C}$ is convex, (2.1) is a convex optimization problem. The following examples give an intuition by showing that the setting described in this section generalizes many important cases previously regarded in the literature. For a (finite) set $I$, we define the coordinate subspace $\mathcal{E}_{I}:=\left\{y \in \mathcal{E}: y_{i}=0 \forall i \notin I\right\}$. Additionally, we denote by $\mathbb{R}^{I}$ the space of elements with real entries indexed by the elements of $I$.

\section{Example 2.3.}

(2.3.1) Recovery of sparse vectors by $\ell_{1}$-minimization

For the recovery of sparse vectors $x \in \mathbb{R}^{n}$, let $\mathcal{X}=\mathcal{E}=\mathcal{C}=\mathbb{R}^{n}, B$ be the identity and $\|\cdot\|=\|\cdot\|_{1}$; then $\mathcal{D}=\mathbb{R}^{n}$. Let $\mathcal{P}$ be the set of orthogonal projectors onto all coordinate 
subspaces of $\mathbb{R}^{n}$, and define $\bar{P}:=I_{n}-P$, where $I_{n}$ denotes the identity mapping on $\mathbb{R}^{n}$. If $S$ is the index set of components on which $P$ projects, then $P B x=P x=x_{S}$, where $x_{S} \in \mathbb{R}^{n}$ equals $x$ on $S$ and is 0 otherwise. Similarly, for the complement $\bar{S}:=[n] \backslash S, \bar{P} B x=x_{\bar{S}}$. Define the nonnegative weight $\nu(P):=\operatorname{rank}(P)$, so that $\nu(P)$ is the number of nonzero components of the subspace $P$ projects onto. The notion of general sparsity reduces to the classical sparsity of nonzero entries in a vector $x \in \mathbb{R}^{n}$ (if $P x=x$, then $\|x\|_{0} \leq \nu(P)$ ), and the recovery problem (2.1) becomes classical $\ell_{1}$-minimization.

(2.3.2) Recovery of sparse nonnegative vectors by $\ell_{1}$-minimization

For the recovery of nonnegative vectors let $\mathcal{X}, \mathcal{E}, B, \mathcal{P}, \nu(P), \bar{P},\|\cdot\|$ be defined as in the previous example, and let $\mathcal{C}=\mathbb{R}_{+}^{n}$, implying $\mathcal{D}=\mathbb{R}_{+}^{n}$. As before, the notion of general sparsity now simplifies to the classical sparsity of nonzero entries in a nonnegative vector $x \in \mathbb{R}_{+}^{n}$, and the recovery problem (2.1) becomes nonnegative $\ell_{1}$-minimization with $P B x=x_{S}$ and $\bar{P} B x=x_{\bar{S}}$.

(2.3.3) Recovery of low-rank matrices by nuclear norm minimization

Let $\mathcal{X}=\mathcal{E}=\mathcal{C}=\mathbb{R}^{n_{1} \times n_{2}}$. Let the representation map $B$ be the identity (thus, $\mathcal{D}=\mathbb{R}^{n_{1} \times n_{2}}$ ), and let the norm $\|\cdot\|$ be the nuclear norm $\|\cdot\|_{*}$. For some positive integer $k$ and a set $I \subseteq[k]$, define the matrix $T_{I}^{k} \in \mathbb{R}^{k \times k}$ to be a matrix with ones on the diagonal at positions $(i, i)$ for $i \in I$ and zeros elsewhere. Let $\mathcal{O}^{k}$ be the set of $k \times k$ orthogonal matrices. Then define the set $\mathcal{P}$ of projections $P: \mathbb{R}^{n_{1} \times n_{2}} \rightarrow \mathbb{R}^{n_{1} \times n_{2}}$ as

$$
\mathcal{P}:=\left\{X \mapsto U T_{I}^{n_{1}} U^{\top} X V T_{I}^{n_{2}} V^{\top}: U \in \mathcal{O}^{n_{1}}, V \in \mathcal{O}^{n_{2}}, I \subseteq\left\{1, \ldots, \min \left\{n_{1}, n_{2}\right\}\right\}\right\}
$$

For $P \in \mathcal{P}$ defined by $U \in \mathcal{O}^{n_{1}}, V \in \mathcal{O}^{n_{2}}$ and index set $I$, define the nonnegative weight $\nu(P)$ as $\nu(P)=|I|$, and $\bar{P}$ as

$$
X \mapsto U\left(I_{n_{1}}-T_{I}^{n_{1}}\right) U^{\top} X V\left(I_{n_{2}}-T_{I}^{n_{2}}\right) V^{\top},
$$

where $I_{n_{i}}$ denotes the identity matrix of size $n_{i}$, so that $I_{n_{i}}-T_{I}^{n_{i}}=T_{\left[n_{i}\right] \backslash I^{1}}^{n_{i}}{ }^{1}$

The intuition behind these projections is as follows. If $U, V$ are chosen such that $X=U \Sigma V^{\top}$ is the singular value decomposition of $X$, then $P$ first projects $X$ onto $\Sigma$ containing the singular values $\sigma_{1}(X) \geq \cdots \geq \sigma_{\min \left\{n_{1}, n_{2}\right\}}(X)$, then sets $\sigma_{i}(X)=0$ for all $i \notin I$ via left- and right-multiplication of $T_{I}^{n_{1}}$ and $T_{I}^{n_{2}}$, respectively, and transforms the resulting diagonal matrix $\tilde{\Sigma}$ back by $U \tilde{\Sigma} V^{\top}$.

A matrix $X \in \mathbb{R}^{n_{1} \times n_{2}}$ is rank-s-sparse, i.e., there exist at most $s$ nonzero singular values, if and only if there exists a projection $P \in \mathcal{P}$ with corresponding index set $I$ with $P X=X$ and $|I| \leq s$. Thus, $\sigma_{i}(X)=0$ for all $i \notin I$, and accordingly, $\operatorname{rank}(X) \leq s$. Therefore, the recovery problem (2.1) becomes low-rank matrix recovery, and sparsity translates to low-rankness.

(2.3.4) Recovery of positive definite low-rank matrices by nuclear norm minimization

For the recovery of positive semidefinite matrices, let $\mathcal{S}_{+}^{n}$ be the set of psd matrices of size $n \times n$. Then consider $\mathcal{X}=\mathcal{E}=\mathcal{S}^{n}, \mathcal{C}=\mathcal{S}_{+}^{n}$, and let $B$ be the identity map (thus, $\left.\mathcal{D}=\mathcal{S}_{+}^{n}\right)$. The definitions of $\mathcal{P}, \bar{P}, \nu(P)$ and $\|\cdot\|$ are as in the previous example. Again,

\footnotetext{
${ }^{1}$ Note that $U T_{I}^{n_{1}} U^{\top} X V T_{I}^{n_{2}} V^{\top}$ and $U\left(I_{n_{1}}-T_{I}^{n_{1}}\right) U^{\top} X V\left(I_{n_{2}}-T_{I}^{n_{2}}\right) V^{\top}$ denote matrix products, since $U, T_{I}^{n_{1}}, T_{I}^{n_{2}} X$, and $V$ are matrices, and not linear maps.
} 
the notion of sparsity simplifies to low-rankness. Recovery problem (2.1) becomes lowrank recovery for positive semidefinite matrices.

Examples with a nontrivial representation map $B: \mathcal{X} \rightarrow \mathcal{E}$ are given by settings in which the vectors or matrices obey a certain block-structure or block-diagonal form, even with overlapping blocks. These settings are not as well studied as the settings described above, so that they are discussed in the subsequent sections in more detail.

In order to characterize the cases in which a sparse element $x^{(0)}$ can be recovered from its measurements $b=A x^{(0)}$ using the recovery problem (2.1), we consider the following assumptions on the sets $\mathcal{C}, \mathcal{D}, \mathcal{P}$ and the norm $\|\cdot\|$.

(A1) For every $P \in \mathcal{P}$ it holds that ○ $P^{2}=P$, i.e., $P$ is a projector, and

$\circ P y \in \mathcal{D}$ for all $y \in \mathcal{D}$.

Moreover, $B: \mathcal{X} \rightarrow \mathcal{E}$ is injective, and for all $c_{1}, c_{2} \in \mathcal{C}, c_{1}+c_{2} \in \mathcal{C}$ holds.

(A2) For every $P \in \mathcal{P}$ the corresponding linear map $\bar{P}: \mathcal{E} \rightarrow \mathcal{E}$ satisfies

$\circ \bar{P} P=0$, and

$\circ \bar{P} y \in \mathcal{D}$ for all $y \in \mathcal{D}$.

(A3) For all $y \in \mathcal{E}$ and all $P \in \mathcal{P}$ it holds that $y=P y+\bar{P} y$.

(A4a) For all $s \geq 0, P \in \mathcal{P}_{s}$, for all $x, z \in \mathcal{C}$ with $P B x=B x$ (i.e., $x$ being $s$-sparse) and $v:=x-z$ and all $v^{(1)}, v^{(2)} \in \mathcal{C}$ with $v=v^{(1)}-v^{(2)}$ it holds that

$$
\|B x\| \leq\|B z\|+\left\|P B v^{(1)}\right\|-\left\|P B v^{(2)}\right\|-\|\bar{P} B v\| .
$$

(A4b) For all $s \geq 0, P \in \mathcal{P}_{s}$, for all $x, z \in \mathcal{C}$ with $P B x=B x$ (i.e., $x$ being $s$-sparse) and $v:=x-z$ there exist $v^{(1)}, v^{(2)} \in \mathcal{C}$ with $v=v^{(1)}-v^{(2)}$ and

$$
\|B x\| \leq\|B z\|+\left\|P B v^{(1)}\right\|-\left\|P B v^{(2)}\right\|-\|\bar{P} B v\| .
$$

Note that Assumptions (A1)-(A3) are satisfied in the different settings in Example 2.3, because $\mathcal{P}$ consists of orthogonal projections. Only Assumptions (A4a) and (A4b) remain to be verified. A discussion of these assumptions in the settings of Example 2.3 will follow after the main result of this section.

We can now define two versions of a null space property.

Definition 2.4. The linear sensing map $A$ satisfies the general null space property of type $I$ of order $s$ for the set $\mathcal{C}$ if and only if for all $v \in(\mathcal{N}(A) \cap(\mathcal{C}+(-\mathcal{C})))$ with $B v \neq 0$ and all $P \in \mathcal{P}_{s}$ it holds that

$$
\begin{aligned}
-\bar{P} B v \in \mathcal{D} \Longrightarrow & \exists v^{(1)}, v^{(2)} \in \mathcal{C} \text { with } v=v^{(1)}-v^{(2)} \text { and } \\
& \left\|P B v^{(1)}\right\|-\left\|P B v^{(2)}\right\|<\|\bar{P} B v\|,
\end{aligned}
$$

where $\mathcal{N}(A):=\{v \in \mathcal{X}: A v=0\}$ is the null space of the linear sensing map $A$.

Definition 2.5. The linear sensing map $A$ satisfies the general null space property of type $I I$ of order $s$ for the set $\mathcal{C}$ if and only if for all $v \in(\mathcal{N}(A) \cap(\mathcal{C}+(-\mathcal{C})))$ with $B v \neq 0$ and all $P \in \mathcal{P}_{s}$ it holds that

$$
\begin{aligned}
&-\bar{P} B v \in \mathcal{D} \Longrightarrow \Rightarrow v^{(1)}, v^{(2)} \in \mathcal{C} \text { with } v=v^{(1)}-v^{(2)}: \\
&\left\|P B v^{(1)}\right\|-\left\|P B v^{(2)}\right\|<\|\bar{P} B v\| .
\end{aligned}
$$


Remark 2.6. We emphasize that $\left(\mathrm{NSP}_{-} \mathrm{I}^{\mathcal{C}}\right)$ and $\left(\mathrm{NSP}-\mathrm{II}^{\mathcal{C}}\right)$ only differ in the quantifiers (namely, $\exists v^{(1)}, v^{(2)}$ versus $\left.\forall v^{(1)}, v^{(2)}\right)$, and these are closely connected to the quantifiers in $(\mathrm{A} 4 \mathrm{a})$ and $(\mathrm{A} 4 \mathrm{~b})$ : If

$$
\|B x\| \leq\|B z\|+\left\|P B v^{(1)}\right\|-\left\|P B v^{(2)}\right\|-\|\bar{P} B v\|
$$

is satisfied for all $v^{(1)}, v^{(2)} \in \mathcal{C}$ with $v=v^{(1)}-v^{(2)}$, there only need to exist $v^{(1)}, v^{(2)} \in \mathcal{C}$ with $v=v^{(1)}-v^{(2)}$ such that

$$
\left\|P B v^{(1)}\right\|-\left\|P B v^{(2)}\right\|-\|\bar{P} B v\|<0
$$

holds (if $-\bar{P} B v \in \mathcal{D}$ ), which is $\left(\mathrm{NSP}_{\mathrm{I}}{ }^{\mathcal{C}}\right.$ ). Otherwise, if there only exist $v^{(1)}, v^{(2)} \in \mathcal{C}$ with $v=v^{(1)}-v^{(2)}$ such that $(2.2)$ holds, then $(2.3)$ must be satisfied by all $v^{(1)}, v^{(2)} \in \mathcal{C}$ with $v=v^{(1)}-v^{(2)}$, which is $\left(\mathrm{NSP}-\mathrm{II}^{\mathcal{C}}\right)$. As we will see in the subsequent theorem, under Assumption (A4a), $\left(\mathrm{NSP}_{-} \mathrm{I}^{\mathcal{C}}\right)$ characterizes uniform recovery, and if Assumption (A4b) is satisfied, then $\left(\mathrm{NSP}-\mathrm{II}^{\mathcal{C}}\right)$ is needed for uniform recovery.

The reason for formulating these two slightly different null space properties is that in the settings described in Examples 2.3, the unrestricted cases, i.e., $\mathcal{C}=\mathcal{X}$, satisfy Assumption (A4b) and thus need (2.5) for uniform recovery, whereas in the restricted cases, Assumption (A4a) holds, so that $\left(\mathrm{NSP}_{-} \mathrm{I}^{\mathcal{C}}\right)$ suffices for uniform recovery.

The following main result of this section states that the above defined null space properties $\left(\mathrm{NSP}-\mathrm{II}^{\mathcal{C}}\right)$ and $\left(\mathrm{NSP}-\mathrm{I}^{\mathcal{C}}\right)$ exactly characterize the uniform recovery of a sufficiently sparse $x \in \mathcal{C}$ from its measurements $b=A x$ using (2.1), depending on which assumptions are satisfied.

Theorem 2.7. Suppose that Assumptions (A1), (A2) and (A3) are satisfied. Let A be a linear sensing map and $s \geq 1$.

(1) If Assumption (A4a) is satisfied, then the following statements are equivalent:

(i) Every s-sparse $x^{(0)} \in \mathcal{C}$ is the unique solution of (2.1) with $b=A x^{(0)}$.

(ii) A satisfies the general null space property $\left(\mathrm{NSP}-\mathrm{I}^{\mathcal{C}}\right)$ of order $s$ for the set $\mathcal{C}$.

(2) If Assumption (A4b) is satisfied, then the following statements are equivalent:

(i) Every s-sparse $x^{(0)} \in \mathcal{C}$ is the unique solution of (2.1) with $b=A x^{(0)}$.

(ii) A satisfies the general null space property $\left(\mathrm{NSP}-\mathrm{II}^{\mathcal{C}}\right)$ of order $s$ for the set $\mathcal{C}$.

Proof. For the first equivalence, let $s \geq 1$ and suppose Assumptions (A1)-(A3) and (A4a) are satisfied.

Assume that if $A x=b$ has an $s$-sparse solution $x^{(0)} \in \mathcal{C}$, then $x^{(0)}$ is the unique solution of (2.1). Let $P \in \mathcal{P}_{s}$ and $v \in(\mathcal{N}(A) \cap(\mathcal{C}+(-\mathcal{C})))$ with $B v \neq 0$ and $-\bar{P} B v \in \mathcal{D}$. Since $v \in \mathcal{C}+(-\mathcal{C})$, there exist $v^{(1)}, v^{(2)} \in \mathcal{C}$ with $v=v^{(1)}-v^{(2)}$. Define

$$
w_{s}^{(1)}:=P B v^{(1)}, \quad w_{s}^{(2)}:=P B v^{(2)}, \quad w_{\bar{s}}:=\bar{P} B v .
$$

Since by Assumption (A1), $P B x \in \mathcal{D}$ for all $x \in \mathcal{C}$, there exist $v_{s}^{(1)}, v_{s}^{(2)}, v_{\bar{s}} \in \mathcal{C}$ with $B v_{s}^{(1)}=w_{s}^{(1)}, B v_{s}^{(2)}=w_{s}^{(2)}$ and $B v_{\bar{s}}=w_{\bar{s}}$. Due to Assumption (A3)

$$
\begin{aligned}
B v=P B v+\bar{P} B v=P B v^{(1)}-P B v^{(2)}+\bar{P} B v & =B v_{s}^{(1)}-B v_{s}^{(2)}+B v_{\bar{s}} \\
& =B\left(v_{s}^{(1)}-v_{s}^{(2)}+v_{\bar{s}}\right),
\end{aligned}
$$


which implies $v=v_{s}^{(1)}-v_{s}^{(2)}+v_{\bar{s}}$, since $B$ is injective by Assumption (A1). Accordingly,

$$
0=A v=A\left(v_{s}^{(1)}-v_{s}^{(2)}+v_{\bar{s}}\right) \quad \Leftrightarrow \quad A\left(v_{s}^{(2)}-v_{\bar{s}}\right)=A v_{s}^{(1)} .
$$

By Assumption (A1), $P B v_{s}^{(1)}=P w_{s}^{(1)}=P P B v^{(1)}=P B v^{(1)}=w_{s}^{(1)}=B v_{s}^{(1)}$, i.e., $v_{s}^{(1)}$ is $s$-sparse. Moreover, by Assumption (A1), $P y \in \mathcal{D}$ for all $y \in \mathcal{D}, B$ is injective and $c_{1}+c_{2} \in \mathcal{C}$ for all $c_{1}, c_{2} \in \mathcal{C}$. Thus, $v^{(1)} \in \mathcal{C}$ implies $B v_{s}^{(1)}=w_{s}^{(1)}=P B v^{(1)} \in \mathcal{D}$. Since $\mathcal{D}=\{B x: x \in \mathcal{C}\}$, there exists $u \in \mathcal{C}$ with $B u=B v_{s}^{(1)} \in \mathcal{D}$. This in turn implies $v_{s}^{(1)}=u \in \mathcal{C}$, since $B$ is injective. Analogously, $v_{s}^{(2)} \in \mathcal{C}$ and $-v_{\bar{s}} \in \mathcal{C}$, since $v^{(2)} \in \mathcal{C}$ and $-\bar{P} B v \in \mathcal{D}$ per assumption. Altogether, this yields $v_{s}^{(2)}-v_{\bar{s}} \in \mathcal{C}$, since $c_{1}+c_{2} \in \mathcal{C}$ for all $c_{1}, c_{2} \in \mathcal{C}$. The uniqueness property of $A$ for the $s$-sparse $v_{s}^{(1)}$ now implies

$$
\begin{aligned}
& \left\|B v_{s}^{(1)}\right\|<\left\|B v_{s}^{(2)}-B v_{\bar{s}}\right\| \leq\left\|B v_{s}^{(2)}\right\|+\left\|B v_{\bar{s}}\right\| \\
\Leftrightarrow & \left\|B v_{s}^{(1)}\right\|-\left\|B v_{s}^{(2)}\right\|-\left\|B v_{\bar{s}}\right\|<0 \\
\Leftrightarrow & \left\|P B v^{(1)}\right\|-\left\|P B v^{(2)}\right\|-\|\bar{P} B v\|<0 .
\end{aligned}
$$

For the reverse direction, assume $A$ satisfies the general null space property $\left(\mathrm{NSP}-\mathrm{I}^{\mathcal{C}}\right)$ of order $s$ for the set $\mathcal{C}$. Let $x, z \in \mathcal{C}$ with $B x \neq B z, A x=A z$ and $x$ being $s$-sparse, i.e., there exists $P \in \mathcal{P}_{s}$ with $P B x=B x$. Define $v:=x-z \in \mathcal{N}(A) \cap(\mathcal{C}+(-\mathcal{C}))$ with $-\bar{P} B v=-\bar{P} B x+\bar{P} B z=-\bar{P} P B x+\bar{P} B z=\bar{P} B z \in \mathcal{D}$, since $\bar{P} P=0$ and $\bar{P} y \in \mathcal{D}$ for all $y \in \mathcal{D}$ (Assumption (A2)). The general null space property (NSP-I ${ }^{\mathcal{C}}$ ) implies the existence of $v^{(1)}, v^{(2)} \in \mathcal{C}$ with $v=v^{(1)}-v^{(2)}$ and

$$
\left\|P B v^{(1)}\right\|-\left\|P B v^{(2)}\right\|-\|\bar{P} B v\|<0 .
$$

Together with Assumption (A4a) this yields

$$
\|B x\| \leq\|B z\|+\left\|P B v^{(1)}\right\|-\left\|P B v^{(2)}\right\|-\|\bar{P} B v\|<\|B z\| .
$$

This shows that $x$ must be the unique solution of (2.1), which completes the proof of the first equivalence.

For the second equivalence, note that the forward direction implying the general null space property $\left(\mathrm{NSP}-\mathrm{II}^{\mathcal{C}}\right)$ is completely analogous to above. The proof for the reverse direction needs one small adjustment. In this case, the general null space property $\left(\mathrm{NSP}-\mathrm{II}^{\mathcal{C}}\right)$ implies that (2.4) holds for all $v^{(1)}, v^{(2)} \in \mathcal{C}$ with $v=v^{(1)}-v^{(2)}$. Since $v \in \mathcal{C}+(-\mathcal{C})$, there exists at least one decomposition $v=v^{(1)}-v^{(2)}$ with $v^{(1)}, v^{(2)} \in \mathcal{C}$. Together with Assumption (A4b) this implies

$$
\|B x\| \leq\|B z\|+\left\|P B v^{(1)}\right\|-\left\|P B v^{(2)}\right\|-\|\bar{P} B v\|<\|B z\|,
$$

which concludes the proof of the second equivalence.

Remark 2.8. Let $\mathcal{C}=\mathcal{X}$ and $\mathcal{D}=\mathcal{E}$. Then our setting simplifies to the framework in [24]. Clearly, under Assumptions A.1-A.3 in [24], Assumptions (A1), (A2) and (A4b) are satisfied. In this case, $\left(\mathrm{NSP}-\mathrm{II}^{\mathcal{C}}\right)$ is only a sufficient condition, and it implies the sufficient condition in [24, Lemma 3.1], namely

$$
\|P B v\|<\|\bar{P} B v\|
$$


for all $P \in \mathcal{P}_{s}$ and all $v \in \mathcal{N}(A), B v \neq 0$. If additionally Assumption (A3) holds, then $(2.5)$ and $\left(\mathrm{NSP}-\mathrm{II}^{\mathcal{C}}\right)$ are also necessary conditions and in fact equivalent.

For all the settings derived in Example 2.3, specific NSPs are already known in the literature. In the next example, we demonstrate how these NSPs emerge from the generalized null space properties $\left(\mathrm{NSP}_{-} \mathrm{I}^{\mathcal{C}}\right)$ and $\left(\mathrm{NSP}-\mathrm{II}^{\mathcal{C}}\right)$. As already mentioned before, Assumptions (A1)-(A3) are satisfied in all four settings of Example 2.3. In case that the NSPs are satisfied, the null space characterizations provide algorithmically tractable algorithms to find the solution, using linear programming, semidefinite programming or the convex optimization problem of minimizing the nuclear norm, respectively. However, already in the special case of recovery of sparse vectors, it is $\mathcal{N} \mathcal{P}$-hard to check whether a given sensing matrix $A$ satisfies the classical null space property [45].

\section{Example 2.9.}

(2.9.1) Recovery of sparse vectors by $\ell_{1}$-minimization, Example (2.3.1) continued

The following example shows that Assumption (A4a) is violated. Let $z=(2,0,0)^{\top}$, $x=(0,-1,0)^{\top}$ so that $v=x-z=(-2,-1,0)^{\top}$. Let $P$ be the projection onto the first two coordinates. The decomposition $v^{(1)}=(8,9,0)^{\top}$ and $v^{(2)}=(10,10,0)^{\top}$ yields

$$
\|B x\|_{1}=1>2+17-20-0=\|B z\|_{1}+\left\|P B v^{(1)}\right\|_{1}-\left\|P B v^{(2)}\right\|_{1}-\|\bar{P} B v\|_{1} .
$$

However, Assumption (A4b) is satisfied, so that $\left(\mathrm{NSP}-\mathrm{II}^{\mathcal{C}}\right)$ characterizes uniform recovery. For the decomposition $v=v-0$, where 0 denotes the all-zero vector, condition $\left(\mathrm{NSP}-\mathrm{II}^{\mathcal{C}}\right.$ ) simplifies to the regular null space property (see, e.g., [18]):

$$
\left\|v_{S}\right\|_{1}<\left\|v_{\bar{S}}\right\|_{1} \quad \forall v \in \mathcal{N}(A) \backslash\{0\}, \forall S \subseteq[n],|S| \leq s,
$$

where $S$ denotes the index set of components on which $P$ projects, and $\bar{S}:=[n] \backslash S$. It can be shown that Condition $\left(\mathrm{NSP}-\mathrm{II}^{\mathcal{C}}\right)$ and $\left\|v_{S}\right\|_{1}<\left\|v_{\bar{S}}\right\|_{1}$ are equivalent.

(2.9.2) Recovery of sparse nonnegative vectors by $\ell_{1}$-minimization, Example (2.3.2) continued

In contrast to the previous setting, Assumption (A4a) is satisfied for the recovery of sparse nonnegative vectors. It can be shown that the general null space property $\left(\mathrm{NSP}-\mathrm{II}^{\mathcal{C}}\right)$ of order $s$ for the set $\mathcal{C}$ is equivalent to the known nonnegative null space property [28, 47]:

$$
v_{\bar{S}} \leq 0 \Longrightarrow \sum_{i \in S} v_{i}<\left\|v_{\bar{S}}\right\|_{1}, \forall v \in \mathcal{N}(A) \backslash\{0\}, \forall S \subseteq[n],|S| \leq s, \quad\left(\mathrm{NSP}_{\geq 0}\right)
$$

where again $S$ denotes the index set of components on which $P$ projects.

(2.9.3) Recovery of low-rank matrices by nuclear norm minimization, Example (2.3.3) continued

Since vectors can be interpreted as diagonal matrices, the same counterexample as Example (2.9.1) shows that Assumption (A4a) is not fulfilled. However, it can be shown that Assumption (A4b) is satisfied, so that the general null space property (NSP-II ${ }^{\mathcal{C}}$ ) characterizes uniform recovery for low-rank matrix matrices. Using the decomposition 
$V=V-0$, condition $\left(\mathrm{NSP}-\mathrm{II}^{\mathcal{C}}\right)$ simplifies to the well-known null space property $[38,40]$, [18, Theorem 4.40]:

$$
\sum_{j \in S} \sigma_{j}(V)<\sum_{j \in \bar{S}} \sigma_{j}(V), \forall V \in \mathcal{N}(A) \backslash\{0\}, \forall S \subseteq\left[\min \left\{n_{1}, n_{2}\right\}\right],|S| \leq s,
$$

where $\sigma(V)$ is the vector of singular values of $V$, and $S$ is connected to a projection $P \in \mathcal{P}$ with index set $I$ by $S=I$. For symmetric matrices $X \in \mathcal{S}^{n}$ this simplifies to

$$
\left\|\lambda_{S}(V)\right\|_{1}<\left\|\lambda_{\bar{S}}(V)\right\|_{1}, \forall V \in \mathcal{N}(A) \backslash\{0\}, \forall S \subseteq[n],|S| \leq s,
$$

where $\lambda(V)$ is the vector of eigenvalues of $V$.

(2.9.4) Recovery of positive semidefinite low-rank matrices by nuclear norm minimization, Example (2.3.4) continued

Again, in contrast to the previous setting, Assumption (A4a) is satisfied for recovery of positive semidefinite low-rank matrices. The general null space property $\left(\mathrm{NSP}-\mathrm{I}^{\mathcal{C}}\right.$ ) simplifies to the following null space property [29, 38]:

$$
\begin{aligned}
\lambda_{\bar{S}}(V) & \leq 0 \Longrightarrow \sum_{j \in S} \lambda_{j}(V)<\left\|\lambda_{\bar{S}}(V)\right\|_{1}, \\
\forall V & \in\left(\mathcal{N}(A) \cap \mathcal{S}^{n}\right) \backslash\{0\}, \forall S \subseteq[n],|S| \leq s,
\end{aligned}
$$

where $\lambda(V)$ is the vector of eigenvalues of $V$.

Remark 2.10. The formulation of the nonnegative null space property $\left(\mathrm{NSP}_{\geq 0}\right)$ in Example (2.9.2) already indicates that $\left(\mathrm{NSP}_{\geq 0}\right)$ is weaker than (NSP), since for the left hand side $\sum_{j \in S} v_{j} \leq\left\|v_{s}\right\|_{1}$ holds, and additionally, if the condition $v_{\bar{S}} \leq 0$ for all $v \in \mathcal{N}(A) \backslash\{0\}$ is violated, then the inequality $\sum_{i \in S} v_{i}<\left\|v_{\bar{S}}\right\|_{1}$ need not hold, see Example 4.4 for an explicit case.

Remark 2.11. From the viewpoint of an ordered vector space, the condition in (NSP-I ${ }^{\mathcal{C}}$ ) can be interpreted as follows: Let $(V, \leq)$ be a finite-dimensional ordered real vector space, i.e., a finite-dimensional real vector space $V$ with a partial order $\leq$. The positive cone

$$
C_{V}:=\{x \in V: x \geq 0\}
$$

is a convex cone with $C_{V} \cap\left(-C_{V}\right)=\{0\}$. If $C_{V}$ is full-dimensional (which is the case, for instance for $\mathbb{R}^{n}$ with the usual ordering on vectors, or for the space of symmetric real $n \times n$-matrices with the usual Löwner partial order: $A \preceq B: \Longleftrightarrow B-A \succeq 0$ ), we have $C_{V}-C_{V}=V$ due to the following lemma.

Lemma 2.12. Let $K \subseteq \mathbb{R}^{n}$ be a convex cone. Then $K-K=\mathbb{R}^{n}$ if and only if $K$ is full-dimensional.

Proof. Clearly, if $K$ is not full-dimensional, then $K-K$ is not full-dimensional. For the converse direction see, e.g., Ahmadi and Hall [1, Lemma 1].

Thus, if $\mathcal{C}=C_{V}$ is full-dimensional in $\mathbb{R}^{n}$, the null space conditions (NSP-I ${ }^{\mathcal{C}}$ ) and $\left(\mathrm{NSP}-\mathrm{II}^{\mathcal{C}}\right)$ simplify a bit: the requirement $v \in \mathcal{N}(A) \cap(\mathcal{C}+(-\mathcal{C})$ ) can be replaced by $v \in \mathcal{N}(A)$. Moreover, the decomposition $v=v^{(1)}-v^{(2)}$ with $v^{(1)}, v^{(2)} \in \mathcal{C}$ always exists. 
Remark 2.13. Our setting also captures the constraint that $x$ is known to be boxconstrained and $x \in \mathbb{Z}^{n}$. For $\ell, u \in \mathbb{Z}^{n}$ define $[\ell, u]_{\mathbb{Z}}:=\left\{x \in \mathbb{Z}^{n}: \ell \leq x \leq u\right\}$ to model lower- and upper bound constraints of $x \in \mathbb{Z}^{n}$, where $\leq$ is meant component-wise. Let $\mathcal{X}=\mathcal{E}=\mathbb{R}^{n}, \mathcal{C}=[\ell, u]_{\mathbb{Z}}$ with $\ell \leq 0 \leq u$. Let $B$ be the identity map, so that $\mathcal{D}=\mathcal{C}$. Furthermore, let $\mathcal{P}$ be the set of orthogonal projectors onto all coordinate subspaces of $\mathbb{R}^{n}$, and define $\bar{P}=I_{n}-P$, where $I_{n}$ denotes the identity mapping on $\mathbb{R}^{n}$. Define the nonnegative weight $\nu(P):=\operatorname{rank}(P)$, so that $\nu(P)$ is the number of nonzero components of the subspace on which $P$ projects. This yields the recovery problem

$$
\min \left\{\|x\|_{1}: A x=b, x \in[\ell, u]_{\mathbb{Z}}\right\},
$$

which has been considered in, e.g., [26, 30]. Due to the box-constraints $\ell \leq x \leq u$, the last part of Assumption (A1), $c_{1}+c_{2} \in \mathcal{C}$ for all $c_{1}, c_{2} \in \mathcal{C}$, is no longer satisfied in general. However, an inspection of the proof of Theorem 2.7 reveals that the condition

$$
P B c_{1}, \bar{P} B c_{2} \in \mathcal{D} \Longrightarrow P B c_{1}+\bar{P} B c_{2} \in \mathcal{D}
$$

for all $c_{1}, c_{2} \in \mathcal{C}+(-\mathcal{C})$ suffices. The remaining parts of Assumptions (A1)-(A3) as well as Assumption (A4b) can be proven to be satisfied, whereas the counterexample from Example (2.9.1) shows that Assumption (A4a) is violated in general. Thus, by the second part of Theorem 2.7, the null space property $\left(\mathrm{NSP}-\mathrm{II}^{\mathcal{C}}\right)$ characterizes uniform recovery for box-constrained integer vectors.

Since we are especially interested in recovery for (semidefinite) block-diagonal systems, the next section discusses this setting in detail. More specifically, this setting is derived from the general setup described above, and the specific null space properties for characterizing uniform recovery obtained from $\left(\mathrm{NSP}-\mathrm{I}^{\mathcal{C}}\right)$ as well as $\left(\mathrm{NSP}-\mathrm{II}^{\mathcal{C}}\right)$ are stated.

\section{Semidefinite BLOCK-SYSTEMS}

Define $\mathcal{X}=\mathcal{S}^{n}$. As linear sensing map consider the linear operator $A: \mathcal{S}^{n} \rightarrow \mathbb{R}^{m}$ given by

$$
A(X)=\left(A_{1} \bullet X, \ldots, A_{m} \bullet X\right)^{\top},
$$

where $A_{1}, \ldots, A_{m} \in \mathcal{S}^{n}, b \in \mathbb{R}^{m}$, and $X \in \mathcal{S}^{n}$. The corresponding matrix equation is then $A(X)=b$.

Remark 3.1. Note that in this section, we do not follow the notation of the previous section and denote the image of some linear map $F$ as $F(X)$ in order to avoid confusion with matrix products.

The block-diagonal form can now be defined as follows.

Definition 3.2. Let $k \geq 1$ and $B_{1}, \ldots, B_{k} \neq \varnothing$ a partition of the set $[n]$, i.e., $\bigcup_{i=1}^{n} B_{i}=[n]$ with pairwise disjoint blocks $B_{i}$. A linear operator $A(X)$ is in block-diagonal form with blocks $B_{1}, \ldots, B_{k}$ if and only if $\left(A_{i}\right)_{s, t}=0$ for all $(s, t) \notin\left(B_{1} \times B_{1}\right) \cup \cdots \cup\left(B_{k} \times B_{k}\right)$ and all $i \in[m]$.

For an index set $I \subseteq[n]$ and a matrix $X \in \mathcal{S}^{n}$, write $X_{I}$ for the submatrix containing rows and columns of $X$ indexed by $I$, and write $\mathcal{S}^{I}$ (and $\mathcal{S}_{+}^{I}$ ) as the space of symmetric 
(positive semidefinite) $|I| \times|I|$ matrices with rows and columns indexed by the elements of $I$.

Let $\mathcal{E}=\mathcal{S}^{B_{1}} \times \cdots \times \mathcal{S}^{B_{k}}$. We write $X \in \mathcal{E}$ as

$$
X=\left(\begin{array}{ccc}
X_{B_{1}} & & \\
& \ddots & \\
& & X_{B_{k}}
\end{array}\right) \text { with } X_{B_{i}} \in \mathcal{S}^{B_{i}} \text { for all } i \in[k] .
$$

The representation map $B: \mathcal{X} \rightarrow \mathcal{E}$ takes $X \in \mathcal{X}=\mathcal{S}^{n}$ and generates $\left(X_{B_{1}}, \ldots, X_{B_{k}}\right)^{\top}$ defined as $X_{B_{i}}:=\left\{\left(X_{r s}\right)_{r, s \in B_{i}}\right\}, i \in[k]$; note that entries outside of the blocks are ignored.

The sparsity-induced projections are defined as $\mathcal{P}=\left\{P_{I}: I \subseteq[k]\right\}$, where $P_{I}: \mathcal{E} \rightarrow \mathcal{E}$ is the orthogonal projection onto the subspace $\mathcal{E}_{I}:=\left\{X \in \mathcal{E}: X_{B_{i}}=0 \forall i \notin I\right\}$. The nonnegative weight of a projection $P_{I} \in \mathcal{P}$ is defined as $\nu(P)=|I|$, and $\bar{P}:=P_{[k] \backslash I}$. Finally, let the norm $\|\cdot\|$ be the mixed $*, 1$-norm

$$
\|X\|_{*, 1}:=\sum_{i=1}^{k}\left\|X_{B_{i}}\right\|_{*}
$$

where $\|\cdot\|_{*}$ is the nuclear norm on $\mathcal{S}^{B_{i}}$. An element $X \in \mathcal{X}$ is s-block-sparse, if and only if there exists an index set

$$
I \subseteq[k] \text { with }|I| \leq s \text { and } P_{I}(B(X))=B(X),
$$

which implies that $X_{B_{i}}=0$ for all $i \notin I$. Thus, we obtain a block-sparsity setting for matrices. An important side constraint on the matrix $X$ which is to be recovered is given by $X \succeq 0$. In order to model this side constraint in the general setting from Section 2.1, let $\mathcal{C}=\mathcal{S}_{+}^{n}$ and thus $\mathcal{D}=\mathcal{S}_{+}^{B_{1}} \times \cdots \times \mathcal{S}_{+}^{B_{k}}$. In this case, the general recovery problem (2.1) simplifies to the following convex optimization problem.

$$
\min \left\{\|X\|_{*, 1}: A(X)=b, X \succeq 0\right\} .
$$

Define $\|x\|_{0}:=|\operatorname{supp}(x)|=\left|\left\{i \in[n]: x_{i} \neq 0\right\}\right|$ to be the number of nonzero entries in a vector $x$. Then the number of nonzero blocks in a block-diagonal matrix $X \in \mathcal{S}^{n}$ can be written as

$$
\|X\|_{*, 0}=\left\|\left(\left\|X_{B_{1}}\right\|_{*}, \ldots,\left\|X_{B_{k}}\right\|_{*}\right)^{\top}\right\|_{0} .
$$

Thus, the problem of finding solutions of $A(X)=b$ with minimal number of nonzero blocks is

$$
\min \left\{\|X\|_{*, 0}: A(X)=b, X \succeq 0\right\} .
$$

Then Problem (3.2) is a convex relaxation of (3.3).

Now we discuss the question when it is possible to recover a block-sparse positive semidefinite matrix $X^{(0)}$ with $\left\|X^{(0)}\right\|_{*, 0} \leq s, s \leq k$, from $b=\mathcal{A}\left(X^{(0)}\right)$ using the convex relaxation (3.2). The next definition provides a null space property which will be proved to characterize uniform recovery using (3.2) in Theorem 3.4. 
Definition 3.3. A linear operator $A(X)$ in block-diagonal form satisfies the semidefinite block-matrix null space property of order $s$ if and only if

$$
V_{B_{i}} \preceq 0 \forall i \in \bar{S} \quad \Longrightarrow \quad \sum_{i \in S} \mathbb{1}^{\top} \lambda\left(V_{B_{i}}\right)<\sum_{i \in \bar{S}}\left\|V_{B_{i}}\right\|_{*} \quad\left(\mathrm{NSP}_{*, 1, \succeq 0}^{*}\right)
$$

holds for all $V \in\left(\mathcal{N}(A) \cap \mathcal{S}^{n}\right) \backslash\{0\}$ and all $S \subseteq[k],|S| \leq s$, where $\bar{S}:=[k] \backslash S$ and $\lambda\left(V_{B_{i}}\right)$ is the vector of eigenvalues of $V_{B_{i}}$.

Theorem 3.4. Let $A(X)$ be a linear operator in block-diagonal form and $s \geq 1$. The following statements are equivalent:

(i) Every $X^{(0)} \in \mathcal{S}_{+}^{n}$ with $\left\|X^{(0)}\right\|_{*, 0} \leq s$ is the unique solution of $(3.5)$ with $b=A\left(X^{(0)}\right)$.

(ii) $A(X)$ satisfies the semidefinite block-matrix null space property of order $s$.

Proof. In the situation described above, using $\mathcal{C}=\mathcal{S}_{+}^{n}, \mathcal{D}=\mathcal{S}_{+}^{B_{1}} \times \cdots \times \mathcal{S}_{+}^{B_{k}}$ and the mixed $\ell_{*, 1}$-norm it is easy to see that Assumptions (A1)-(A3) are satisfied. In order to see that Assumption (A4a) holds, let $P:=P_{S} \in \mathcal{P}_{s}$ be a projection, and consider $V=X-Z$ with $Z, X \in \mathcal{S}_{+}^{n}$ and $P(B(X))=B(X)$. Let $V^{(1)}, V^{(2)} \in \mathcal{S}_{+}^{n}$ be a decomposition $V=V^{(1)}-V^{(2)}$. This yields

$$
\begin{aligned}
& \left\|P\left(B\left(V^{(1)}\right)\right)\right\|_{*, 1}-\left\|P\left(B\left(V^{(2)}\right)\right)\right\|_{*, 1} \\
& =\sum_{i=1}^{n} \lambda_{i}\left(P\left(B\left(V^{(1)}\right)\right)\right)-\sum_{i=1}^{n} \lambda_{i}\left(P\left(B\left(V^{(2)}\right)\right)\right)=\sum_{i=1}^{n} \lambda_{i}(P(B(V))) \\
& =\sum_{i=1}^{n} \lambda_{i}(P(B(X)))-\sum_{i=1}^{n} \lambda_{i}(P(B(Z)))=\|P(B(X))\|_{*, 1}-\|P(B(Z))\|_{*, 1} \\
& =\|B(X)\|_{*, 1}-\|P(B(Z))\|_{*, 1}+\|\bar{P}(B(Z))\|_{*, 1}-\|\bar{P}(B(Z))\|_{*, 1} \\
& =\|B(X)\|_{*, 1}-\|B(Z)\|_{*, 1}+\|\bar{P}(B(Z))\|_{*, 1},
\end{aligned}
$$

and consequently,

$$
\|B(X)\|_{*, 1}=\|B(Z)\|_{*, 1}-\|\bar{P}(B(Z))\|_{*, 1}+\left\|P\left(B\left(V^{(1)}\right)\right)\right\|_{*, 1}-\left\|P\left(B\left(V^{(2)}\right)\right)\right\|_{*, 1},
$$

which shows, in conjunction with $\bar{P}(B(Z))=\bar{P}(B(V)+B(X))=\bar{P}(B(V))$, that Assumption (A4a) is satisfied.

It remains to show that $\left(\mathrm{NSP}-\mathrm{I}^{\mathcal{C}}\right)$ is equivalent to $\left(\mathrm{NSP}_{*, 1, \succeq 0}^{*}\right)$. Therefore, let $S \subseteq[k]$, $|S| \leq s$ and $P=P_{S}$ be fixed. Since

$$
\sum_{i=1}^{n} \lambda_{i}(B(V))=\left\|\lambda_{i}\left(B\left(V^{+}\right)\right)\right\|_{*}-\left\|\lambda_{i}\left(B\left(V^{-}\right)\right)\right\|_{*},
$$

where $V^{+}, V^{-} \in \mathcal{S}_{+}^{n}$ with $B(V)=B\left(V^{+}\right)-B\left(V^{-}\right)$, Condition (NSP $*, 1, \succeq 0$ ) clearly implies $\left(\mathrm{NSP}-\mathrm{I}^{\mathcal{C}}\right.$ ) by choosing $V^{(1)}=V^{+}$and $V^{(2)}=V^{-}$.

For the reverse implication, let again $S \subseteq[k],|S| \leq s$ and $P=P_{S}$ be fixed and let $V \in\left(\mathcal{N}(A) \cap \mathcal{S}^{n}\right)$ with $B(V) \neq 0$ and $\bar{P}(B(V)) \preceq 0$. Due to (NSP-I ${ }^{\mathcal{C}}$, there exist $V^{(1)}$, 
$V^{(2)} \succeq 0$ with $V=V^{(1)}-V^{(2)}$ and $\left\|P\left(B\left(V^{(1)}\right)\right)\right\|_{*, 1}-\left\|P\left(B\left(V^{(2)}\right)\right)\right\|_{*, 1}-\|\bar{P}(B(V))\|_{*, 1}<0$. This implies

$$
\begin{aligned}
0 & >\left\|P\left(B\left(V^{(1)}\right)\right)\right\|_{*, 1}-\left\|P\left(B\left(V^{(2)}\right)\right)\right\|_{*, 1}-\|\bar{P}(B(V))\|_{*, 1} \\
& =\sum_{i=1}^{n} \lambda_{i}\left(P\left(B\left(V^{(1)}\right)\right)\right)-\sum_{i=1}^{n} \lambda_{i}\left(P\left(B\left(V^{(2)}\right)\right)\right)-\sum_{i=1}^{n}\left|\lambda_{i}(\bar{P}(B(V)))\right| \\
& =\sum_{i \in S} \mathbb{1}^{\top} \lambda\left(V_{B_{i}}\right)-\sum_{i \in \bar{S}}\left\|V_{B_{i}}\right\|_{*},
\end{aligned}
$$

which establishes $\left(\mathrm{NSP}_{*, 1, \succeq 0}^{*}\right)$ and by Theorem 2.7 finishes the proof.

In order to model the situation where the additional side constraint $X \succeq 0$ is not present, let $\mathcal{C}=\mathcal{X}=\mathcal{S}^{n}$ and $\mathcal{D}=\mathcal{E}=\mathcal{S}^{B_{1}} \times \cdots \times \mathcal{S}^{B_{k}}$, while $A, B, \mathcal{P}, \overline{\bar{P}}$ and the norm $\|\cdot\|$ are defined as above. In this case, the recovery problems (3.3) and (3.2) become

$$
\begin{aligned}
\min \left\{\|X\|_{*, 0}: A(X)=b, X \in \mathcal{S}^{n}\right\} \\
\text { and } \min \left\{\|X\|_{*, 1}: A(X)=b, X \in \mathcal{S}^{n}\right\},
\end{aligned}
$$

respectively. Note that this setting can be obtained by combining the block/group case and the matrix case in [24].

Definition 3.5. A linear operator $A(X)$ in block-diagonal form satisfies the block-matrix null space property of order $s$ if and only if

$$
\sum_{i \in S}\left\|V_{B_{i}}\right\|_{*}<\sum_{i \in \bar{S}}\left\|V_{B_{i}}\right\|_{*}
$$

holds for all $V \in\left(\mathcal{N}(A) \cap \mathcal{S}^{n}\right) \backslash\{0\}$ and all $S \subseteq[k],|S| \leq s$.

Theorem 3.6. Let $A(X)$ be a linear operator in block-diagonal form and $s \geq 1$. The following statements are equivalent:

(i) Every $X^{(0)} \in \mathcal{S}^{n}$ with $\left\|X^{(0)}\right\|_{*, 0} \leq s$ is the unique solution of $(3.5)$ with $b=A\left(X^{(0)}\right)$.

(ii) $A(X)$ satisfies the block-matrix null space property of order $s$.

As already stated, this result can be obtained from [24] by combining the block and the matrix case. Alternatively, it can be derived from the second part of Theorem 2.7, since it can be shown that Assumption $(\mathrm{A} 4 \mathrm{~b})$ is satisfied. It is then easy to verify that $\left(\mathrm{NSP}-\mathrm{II}^{\mathcal{C}}\right)$ and $\left(\mathrm{NSP}_{*, 1}^{*}\right)$ are equivalent. The following example shows that we cannot apply the first part of Theorem 2.7, since Assumption (A4a) is violated.

Example 3.7. Let $k=2$ and $n=4$ together with the partition [4] $=\{1,2\} \cup\{3,4\}$, that is, blocks $B_{1}=\{1,2\}$ and $B_{2}=\{3,4\}$ and consider

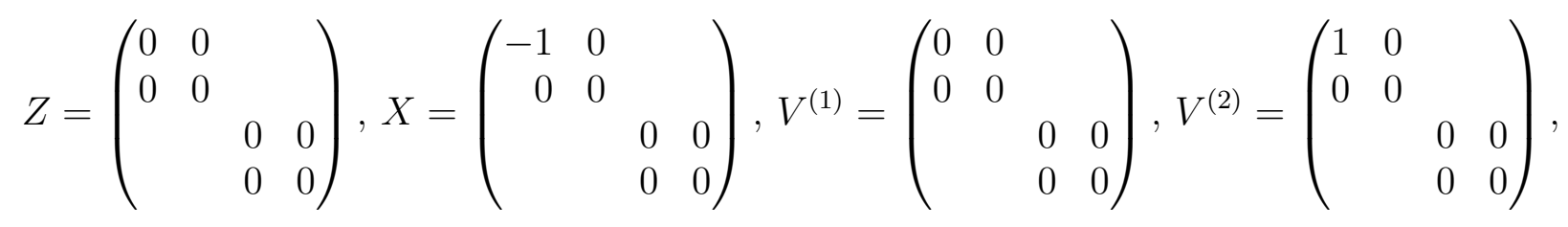


together with $V=X-Z=V^{(1)}-V^{(2)}$. Let $P$ be the projection onto the first block coordinates, i.e., $P=P_{\{1\}} \in \mathcal{P}$. This yields

$$
\begin{aligned}
& \|B(Z)\|_{*, 1}+\left\|P\left(B\left(V^{(1)}\right)\right)\right\|_{*, 1}-\left\|P\left(B\left(V^{(2)}\right)\right)\right\|_{*, 1}-\|\bar{P}(B(V))\|_{*, 1} \\
& =0+0-1-0<1=\|B(X)\|_{*},
\end{aligned}
$$

which is a contradiction to Assumption (A4a).

Remark 3.8. We could also consider $\mathcal{X}=\mathcal{C}=\mathbb{R}^{n_{1} \times n_{2}}$ and possibly overlapping blocks $B_{i} \neq \varnothing$ by $B_{1} \cup \cdots \cup B_{k}=\left[n_{1}\right] \times\left[n_{2}\right]$ instead of a partition $B_{1}, \ldots, B_{k}$ of $[n]$. Additionally, we could replace the inner nuclear norms by arbitrary norms on $\mathbb{R}^{B_{i} \times B_{i}}$. Replacing the inner nuclear norms by the chosen norms $\|\cdot\|$, this also fits in our general setting described in Section 2.1, such that $\left(\mathrm{NSP}_{*, 1}^{*}\right)$ characterizes uniform recovery using

$$
\min \left\{\sum_{i=1}^{k}\left\|X_{B_{i}}\right\|: A(X)=b, X \in \mathbb{R}^{n_{1} \times n_{2}}\right\} .
$$

Note that for block-diagonal matrices we denote by "inner norms" the norms that are applied on each block. This term is also used later in the setting of block-sparse vectors.

\section{INTERRELATIONS BETWEEN THE NSPS AND CLASSIFICATION}

Building upon the general framework, we analyze and classify the relative strengths of the NSPs, in particular for recovery of positive semidefinite block-diagonal matrices and other prominent subclasses, as well as their interrelations. To prepare for this, we also briefly record the NSPs for block-sparse (nonnegative) vectors.

\subsection{Block-SPARSE (NONNEGATIVE) VECTORS}

Since every block-sparse vector $x$ can be interpreted as a block-diagonal matrix $X$ where all blocks are also diagonal matrices, and the entries of $x$ coincide with the eigenvalues of $X$, Theorem 3.4 also yields a characterization for the uniform recovery of block-sparse nonnegative vectors using $\ell_{1,1}$-minimization, which to the best of our knowledge is the first characterization of uniform recovery in this case.

In order to model such block-structured vectors in the general setup of Section 2.1, let $\mathcal{X}=\mathbb{R}^{n}$. The block-structure is now given by a partition $B_{1}, \ldots, B_{k}$ of $[n]$, where each set $B_{i}$ is nonempty. Let $\mathcal{E}=\mathbb{R}^{B_{1}} \times \cdots \times \mathbb{R}^{B_{k}}$, where for a (finite) set $I$, we denote by $\mathbb{R}^{I}$ the space of elements with entries indexed by the elements of the set $I$. We write $y \in \mathcal{E}$ as $y=(y[1], \ldots, y[k])^{\top}$, where $y[i] \in \mathbb{R}^{B_{i}}$ for all $i \in[k]$. In order to model nonnegativity of $x$, let $\mathcal{C}=\mathbb{R}_{+}^{n}$ which yields $\mathcal{D}=\mathbb{R}_{+}^{B_{1}} \times \cdots \times \mathbb{R}_{+}^{B_{k}}$. The representation map $B: \mathcal{X} \rightarrow \mathcal{E}$ maps $x \in \mathcal{C}$ to its block-structured representation $y[i]=\left(x_{j}\right)_{j \in B_{i}}$. The sparsity-induced projections are given by $\mathcal{P}=\left\{P_{I}: I \subseteq[k]\right\}$, where $P_{I}: \mathcal{E} \rightarrow \mathcal{E}$ is the orthogonal projection onto the subspace $\mathcal{E}_{I}:=\{y \in \mathcal{E}: y[i]=0 \forall i \notin I\}$. The nonnegative weight of a projection $P_{I} \in \mathcal{P}$ is defined as $\nu(P)=|I|$, and $\bar{P}:=P_{[k] \backslash I}$. Finally, let the norm $\|\cdot\|$ be the mixed $\ell_{1,1}$-norm $\|x\|_{1,1}:=\sum_{i=1}^{k}\|y[i]\|_{1}$, where $y=B x \in \mathcal{E}$ is the blockstructured representation of $x \in \mathcal{X}$. A vector $x \in \mathcal{X}$ is $s$-block-sparse, if and only if there exists an index set $I \subseteq[k]$ with $|I| \leq s$ and $P_{I} B x=B x$, which for $y=B x$ implies that 
$y[i]=0$ for $i \notin I$. Thus, this represents block-sparsity, and we arrive at the setting of recovery of block-sparse nonnegative vectors. This setting has already been considered in, e.g., [14, 16, 31, 44] without the additional nonnegativity constraint, and in, e.g., [43] for nonnegative block-sparse vectors.

The general recovery problem (2.1) yields the recovery problem

$$
\min \left\{\|x\|_{1,1}: A x=b, x \in \mathbb{R}_{+}^{n}\right\},
$$

which is a convex relaxation of the exact recovery problem

$$
\min \left\{\|x\|_{1,0}: A x=b, x \in \mathbb{R}_{+}^{n}\right\} .
$$

By choosing $A(X)$ in diagonal form, the NSP for nonnegative block-linear systems can be obtained as an immediate corollary of Theorem 3.4. While it is simply a rephrasing of a special case of that theorem in the language of block-sparse vectors, it will help to relate the result further below to the literature of block-linear systems.

Corollary 4.1. Consider a block-linear system $A x=[A[1] \cdots A[k]] x=b$, where $b \in \mathbb{R}^{m}$ and $A \in \mathbb{R}^{m \times n}$ consists of $k$ blocks $A[i] \in \mathbb{R}^{m \times n_{i}}$. The following statements are equivalent:

(i) Every $x^{(0)} \in \mathbb{R}_{+}^{n}$ with $\left\|x^{(0)}\right\|_{1,0} \leq s$ is the unique solution of (4.1) with $b=A x^{(0)}$.

(ii) A satisfies the nonnegative block-linear null space property of order s, i.e.,

$$
v[\bar{S}] \leq 0 \quad \Longrightarrow \quad \sum_{i \in S} \mathbb{1}^{\top} v[i]<\sum_{i \in \bar{S}}\|v[i]\|_{1} \quad\left(\mathrm{NSP}_{1,1, \geq 0}\right)
$$

holds for all $v \in \mathcal{N}(A) \backslash\{0\}$ and all $S \subseteq[k],|S| \leq s$, where $v[\bar{S}]:=(v[i])_{i \in \bar{S}}$.

In order to model the recovery of block-sparse vectors which are not necessarily nonnegative, let $\mathcal{C}=\mathcal{X}=\mathbb{R}^{n}$ and $\mathcal{D}=\mathcal{E}$, while $B, \mathcal{P}, \bar{P}$ are defined as above. This time, we choose the the mixed $\ell_{q, 1}$-norm $\|y\|_{q, 1}:=\sum_{i=1}^{k}\|y[i]\|_{q}$, with $q \geq 1$ on $\mathbb{R}^{B_{i}}$. Note that without the additional constraint $x \geq 0$ it is not necessary to use an inner $\ell_{1}$-norm for recovery. The exact recovery problem using a nonconvex $\ell_{0}$-term is

$$
\min \left\{\|x\|_{q, 0}: A x=b, x \in \mathbb{R}^{n}\right\},
$$

and its convex relaxation reads

$$
\min \left\{\|x\|_{q, 1}: A x=b, x \in \mathbb{R}^{n}\right\} .
$$

Again, we now formulate a null space property, which, by the first part of Theorem 2.7 can be proved to characterize uniform recovery using (4.1).

Similar to the previous section, define the block-linear null space property of order $s$ as

$$
\|v[S]\|_{q, 1}<\|v[\bar{S}]\|_{q, 1}
$$

for all $v \in \mathcal{N}(A) \backslash\{0\}$ and all $S \subseteq[k]$ with $|S| \leq s$, where again $v[S]:=(v[i])_{i \in S}$. This null space property characterizes the recovery for block-linear systems, as will be shown in the subsequent corollary. If the inner $\ell_{q}$-norms are given by the $\ell_{2}$-norm, this characterization is due to Stojnic et al. [44], who state as a remark, that

"it is reasonable to believe that the null-space characterization $[. .$.$] can$ easily be generalized to the $\ell_{p}$ optimization". 
Corollary 4.2. Let $A=[A[1] \cdots A[k]] \in \mathbb{R}^{m \times n}$ be in block-linear form with $k$ blocks, $x=(x[1], \ldots, x[k])^{\top} \in \mathbb{R}^{n}$ and $s \geq 1$. The following statements are equivalent:

(i) Every $x^{(0)} \in \mathbb{R}^{n}$ with $\left\|x^{(0)}\right\|_{q, 0} \leq s$ is the unique solution of (4.3) with $b=A x^{(0)}$.

(ii) A satisfies the block-linear null space property of order s, i.e., $\left(\mathrm{NSP}_{q, 1}\right)$ holds for all $v \in \mathcal{N}(A) \backslash\{0\}$ and all $S \subseteq[n]$ with $|S| \leq s$.

As already stated, Corollary 4.2 directly follows as a special case from Theorem 3.6.

Remark 4.3. Similar to Remark 3.8, we could also consider $\mathcal{X}=\mathcal{C}=\mathbb{R}^{n}$ and possibly overlapping blocks $B_{i} \neq \varnothing$ with $B_{1} \cup \cdots \cup B_{k}=[n]$ instead of a partition $B_{1}, \ldots, B_{k}$ of $[n]$. Additionally, we could replace the inner $\ell_{q}$-norms by arbitrary norms on $\mathbb{R}^{B_{i}}$. Replacing the inner $\ell_{q}$-norms by norms $\|\cdot\|$ also fits in our general setting described in Section 2.1, such that $\left(\mathrm{NSP}_{q, 1}\right)$ characterizes uniform recovery using $\min \left\{\sum_{i=1}^{k}\|x[i]\|: A x=b\right\}$.

TABLE 1. Null space properties for different settings and their references.

\begin{tabular}{|c|c|c|}
\hline Setting & NSP & Reference \\
\hline $\begin{array}{c}\text { Linear case: } \\
\min \left\{\|x\|_{1}: A x=b, x \in \mathbb{R}^{n}\right\}\end{array}$ & $\begin{array}{c}\left\|v_{S}\right\|_{1}<\left\|v_{\bar{S}}\right\|_{1} \\
\forall v \in \mathcal{N}(A) \backslash\{0\}, S \subseteq[n],|S| \leq s\end{array}$ & $\begin{array}{c}{[8,10]} \\
\text { Ex. }(2.9 .1)\end{array}$ \\
\hline $\begin{array}{c}\text { Nonnegative linear case: } \\
\min \left\{\|x\|_{1}: A x=b, x \in \mathbb{R}_{+}^{n}\right\}\end{array}$ & $\begin{array}{c}v_{\bar{S}} \leq 0 \Longrightarrow \sum_{i \in S} v_{i}<\left\|v_{\bar{S}}\right\|_{1} \\
\forall v \in \mathcal{N}(A) \backslash\{0\}, S \subseteq[n],|S| \leq s .\end{array}$ & $\begin{array}{c}{[28,47]} \\
\text { Ex. }(2.9 .2)\end{array}$ \\
\hline $\begin{array}{c}\text { Block-linear case: } \\
\min \left\{\|x\|_{q, 1}: A x=b, x \in \mathbb{R}^{n}\right\}\end{array}$ & $\begin{array}{c}\|v[S]\|_{q, 1}<\|v[\bar{S}]\|_{q, 1} \\
\forall v \in \mathcal{N}(A) \backslash\{0\}, S \subseteq[k],|S| \leq s\end{array}$ & $\begin{array}{c}{[44],} \\
\text { Cor. } 4.2\end{array}$ \\
\hline $\begin{array}{l}\text { Nonnegative block-linear case: } \\
\min \left\{\|x\|_{1,1}: A x=b, x \in \mathbb{R}_{+}^{n}\right\}\end{array}$ & $\begin{array}{l}v[\bar{S}] \leq 0 \Longrightarrow \sum_{i \in S} \mathbb{1}^{\top} v[i]<\|v[\bar{S}]\|_{1,1} \\
\forall v \in \mathcal{N}(A) \backslash\{0\}, S \subseteq[k],|S| \leq s\end{array}$ & Cor. 4.1 \\
\hline $\begin{array}{c}\text { Matrix case: } \\
\min \left\{\|X\|_{*}: A(X)=b, X \in \mathcal{S}^{n}\right\}\end{array}$ & $\begin{array}{c}\left\|\lambda_{S}(V)\right\|_{1}<\left\|\lambda_{\bar{S}}(V)\right\|_{1} \\
\forall V \in \mathcal{N}(A) \backslash\{0\}, S \subseteq[n],|S| \leq s\end{array}$ & $\begin{array}{c}{[38,40]} \\
\text { Ex. }(2.9 .3)\end{array}$ \\
\hline $\begin{array}{c}\text { Semidefinite matrix case: } \\
\min \left\{\|X\|_{*}: A(X)=b, X \in \mathcal{S}_{+}^{n}\right\}\end{array}$ & $\begin{array}{c}\lambda_{\bar{S}}(V) \leq 0 \Longrightarrow \sum_{j \in S} \lambda_{j}(V)<\left\|\lambda_{\bar{S}}(V)\right\|_{1} \\
\forall V \in \mathcal{N}(A) \backslash\{0\}, S \subseteq[n],|S| \leq s\end{array}$ & $\begin{array}{c}{[29,38]} \\
\text { Ex. }(2.9 .4)\end{array}$ \\
\hline $\begin{array}{c}\text { Block-diagonal case: } \\
\min \left\{\|X\|_{*, 1}: A(X)=b, X \in \mathcal{S}^{n}\right\}\end{array}$ & $\begin{array}{c}\sum_{i \in S}\left\|V_{B_{i}}\right\|_{*}<\sum_{i \in \bar{S}}\left\|V_{B_{i}}\right\|_{*} \\
\forall V \in \mathcal{N}(A) \backslash\{0\}, S \subseteq[k],|S| \leq s .\end{array}$ & Thm. 3.6 \\
\hline $\begin{array}{c}\text { Semidefinite block-diagonal case: } \\
\min \left\{\|X\|_{*, 1}: A(X)=b, X \in \mathcal{S}_{+}^{n}\right\}\end{array}$ & $\begin{array}{c}V_{B_{i}} \preceq 0 \forall i \in \bar{S} \\
\Longrightarrow \sum_{i \in S} \mathbb{1}^{\top} \bar{\lambda}\left(V_{B_{i}}\right)<\sum_{i \in \bar{S}}\left\|V_{B_{i}}\right\|_{*} \\
\forall V \in \mathcal{N}(A) \backslash\{0\}, S \subseteq[k],|S| \leq s .\end{array}$ & Thm. 3.4 \\
\hline
\end{tabular}




\subsection{Classification of the Null SpaCe CONDitions}

Table 1 shows the null space properties for many important settings considered in existing literature. If already known, the reference is given in the third column, and if not, the corresponding theorem (resp. corollary) within this paper is stated. Afterwards, we state important relationships between the NSPs for the eight settings considered in Table 1.

Recall from Section 4.1 that the block-linear and the nonnegative block-linear cases are special cases of the block-diagonal and the semidefinite block-diagonal cases. Note however, that the matrix case and the semidefinite matrix cases are not special cases of the block-diagonal and the semidefinite block-diagonal cases (but they still fall into the generalized NSP framework in Theorem 2.7).

With respect to the null space properties in Table 1, we now compare the conditions that need to hold in the cases with and without the additional constraints of the vectors being nonnegative or the matrices being positive semidefinite, when the inner norms used in the respective recovery problems are identical.

Every NSP for a setting where nonnegativity or positive semidefiniteness is present stems from $\left(\mathrm{NSP}-\mathrm{I}^{\mathcal{C}}\right)$ and the NSPs in the other settings can be derived from (NSP-II ${ }^{\mathcal{C}}$, see Section 2. By definition, a linear sensing map which satisfies $\left(\mathrm{NSP}-\mathrm{II}^{\mathcal{C}}\right.$ ) for $\mathcal{C}=\mathcal{C}_{1}$ also satisfies $\left(\mathrm{NSP}-\mathrm{I}^{\mathcal{C}}\right.$ ) for every $\mathcal{C}=\mathcal{C}_{2} \subseteq \mathcal{C}_{1}$, but the converse needs of course not be true. Thus, in the presence of nonnegativity or positive semidefiniteness, the conditions needed for characterizing uniform recovery are not stronger than those needed without this prior knowledge, since $\mathcal{C}_{2}:=\mathbb{R}_{+}^{n} \subseteq \mathbb{R}^{n}=: \mathcal{C}_{1}$ and $\mathcal{C}_{2}:=\mathcal{S}_{+}^{n} \subseteq \mathcal{S}^{n}=: \mathcal{C}_{1}$. The following example shows that exploiting positive semidefiniteness indeed yields a weaker condition for uniform recovery, when using the nuclear norm as inner norm in both cases.

Example 4.4. Let $A_{1}, \ldots, A_{4}$ be the block-diagonal matrices

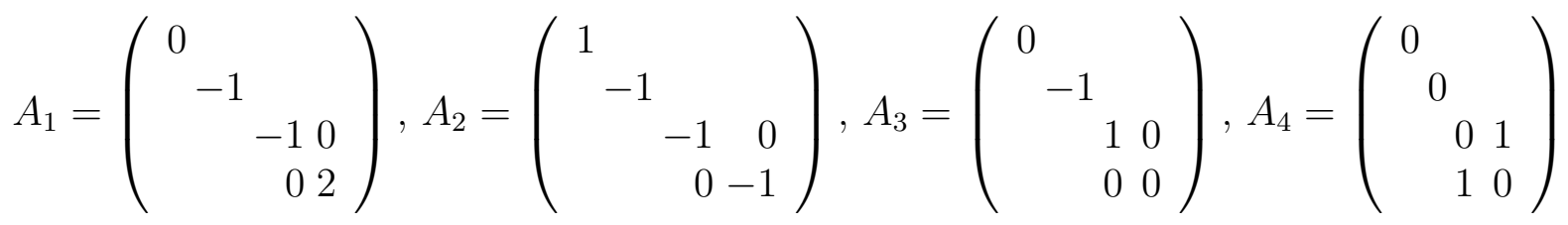

with blocks $B_{1}=\{1\}, B_{2}=\{2\}$ and $B_{3}=\{3,4\}$, and let $b=(-1,0,0,0)^{\top}$. Consider

$$
\min \left\{\|X\|_{*, 0}: A(X)=b, X \succeq 0\right\},
$$

where $A(X)=\left(A_{1} \bullet X, A_{2} \bullet X, A_{3} \bullet X, A_{4} \bullet X\right)^{\top}$, cf. (3.4). In this case, the null space $\mathcal{N}(A)=\left\{V: A_{i} \bullet V=0\right.$ for $\left.i \in[4]\right\}$ consists exactly of the matrices of the form

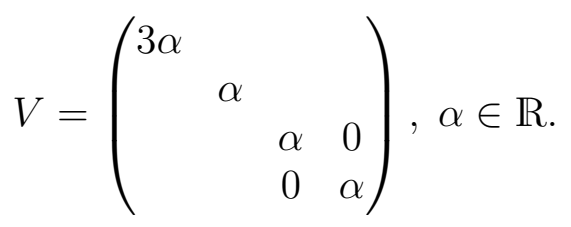

Since only nonzero matrices in the null space of $A$ are of interest for the NSP, $\alpha$ cannot attain the value 0 . The eigenvalues of $V$ are given by $\lambda=(3 \alpha, \alpha, \alpha, \alpha)^{\top}$. For the semidefinite block-matrix null space property of order $s=1$ to hold, the following implications 
stated in Definition 3.3 need to hold for the support sets $S \in\{\varnothing,\{1\},\{2\},\{3\}\}$ :

$$
\begin{aligned}
& S=\varnothing: \quad(3 \alpha, \alpha, \alpha, \alpha)^{\top} \leq 0 \Longrightarrow 0<6|\alpha|, \\
& S=\{1\}: \quad(\alpha, \alpha, \alpha)^{\top} \leq 0 \Longrightarrow 3 \alpha<3|\alpha|, \\
& S=\{2\}: \quad(3 \alpha, \alpha, \alpha)^{\top} \leq 0 \Longrightarrow \alpha<5|\alpha|, \\
& S=\{3\}: \quad(3 \alpha, \alpha)^{\top} \leq 0 \Longrightarrow 2 \alpha<4|\alpha| .
\end{aligned}
$$

These are all satisfied, since for every $V \in \mathcal{N}(A) \backslash\{0\}, \alpha \neq 0$ holds.

However, the block-matrix null space property of order $s$ is violated, since for $S=\{1\}$, $\alpha \neq 0$ it holds that $\sum_{i \in S}\left\|V_{B_{i}}\right\|_{*}=3|\alpha| \geq 3|\alpha|=\sum_{i \in \bar{S}}\left\|V_{B_{i}}\right\|_{*}$, which contradicts (NSP $\left.*, 1\right)$.

This demonstrates that explicitly exploiting nonnegativity or positive semidefiniteness yields stronger results for uniform recovery, which was already indicated in Remark 2.10. In the next subsection, we strengthen this point by explicitly constructing an infinite family of examples that satisfy the nonnegative block-linear null space property $\left(\mathrm{NSP}_{1,1, \geq 0}\right)$. This shows that the proposed null space properties are meaningful in the sense that they are satisfied by certain general (families of) matrices.

\subsection{An INFINITE FAMILY SATISFYING THE NONNEGATIVE BLOCK-LINEAR NSP}

The NSPs for the nonnegative block-linear case and for the semidefinite block-diagonal case hold in many situations. Here, we provide a specific (rather explicit) infinite family of instances to show that even for block sizes $\left(n_{1}, \ldots, n_{k}\right)=(2,1, \ldots, 1)$, the nonnegative block-linear NSP captures cases which are not captured by the (unrestricted) block-linear NSP and which are not captured by the nonnegative linear NSP.

Theorem 4.5. Let $k>m \geq 3$ and $B_{1}, \ldots, B_{k}$ be blocks of sizes $\left(n_{1}, \ldots, n_{k}\right):=(2,1, \ldots, 1)$, and set $n:=\sum_{i=1}^{k} n_{i}=k+1$. There exists an $m \times n$-matrix $A=[A[1] \cdots A[k]]$ such that the nonnegative block-linear NSP (see Corollary 4.1) up to the order $s^{*}:=\lfloor m / 2-1\rfloor$ is satisfied. Moreover, for $m \geq 12$ neither the unrestricted block-linear NSP of order $s^{*}$ is satisfied nor the nonnegative linear NSP of order $s^{*}$ is satisfied.

In the proof of the theorem, we will the apply the following characterization of the nonnegative linear NSP.

Proposition 4.6 (Donoho \& Tanner [11]). Let $A \in \mathbb{R}^{m \times n}$ be a matrix with nonzero columns $a^{(1)}, \ldots, a^{(n)}$ and $m<n$, and let $s \geq 1$. Then $A$ satisfies the nonnegative linear $N S P$ of order $s$ if and only if the polytope $P:=\operatorname{conv}\left\{a^{(1)}, \ldots, a^{(n)}, 0\right\}$ has $n+1$ vertices and is outwardly s-neighborly, that is, every subset of $s$ vertices not including the origin span a face of $P$.

Remark 4.7. With the same preconditions, $A$ satisfies the unrestricted linear NSP of order $s$ if and only if the polytope $P^{\prime}:=\operatorname{conv}\left\{ \pm a^{(1)}, \ldots, \pm a^{(n)}\right\}$ has $2 n$ vertices and is $s$-centrally neighborly, i.e., any $s$ vertices not including an antipodal pair span a face of $P$, see [9, Theorem 1] and also [18, Exer. 4.16]. By results of McMullen and Shephard [33], $P^{\prime}$ can never be $s$-centrally neighborly for $s>\lfloor(m+1) / 3\rfloor$ (see also [12, Section 5.3]). 
Proof of Theorem 4.5. Let $w^{(1)}, \ldots, w^{(k-1)} \in \mathbb{R}^{m-2} \backslash\{0\}$ be $k-1$ distinct points on the moment curve $\left\{\left(t, t^{2}, \ldots, t^{m-2}\right)^{\top}: t \in \mathbb{R}\right\}$ in $\mathbb{R}^{m-2}$. It is well-known that the polytope $P=\operatorname{conv}\left\{w^{(1)}, \ldots, w^{(k-1)}\right\}$ is a cyclic polytope, which is $\lfloor(m-2) / 2\rfloor$-neighborly, see, e.g., [48, Corollary 0.8]. Hence, the nonnegative linear NSP of order $\lfloor(m-2) / 2\rfloor=$ $\lfloor m / 2-1\rfloor$ holds for the matrix $A^{\prime}:=\left[w^{(1)}, \ldots, w^{(k-1)}\right] \in \mathbb{R}^{(m-2) \times(k-1)}$.

Let $p$ be an interior point of $P$ and set $w^{\prime}=(p, 1,0)^{\top}, w^{\prime \prime}=(p, 0,1)^{\top}, \hat{w}^{(i)}=\left(w^{(i)}, 0,0\right)^{\top}$ for $i \in[k-1]$. Let $A:=\left[w^{\prime}, w^{\prime \prime}, \hat{w}^{(1)}, \ldots, \hat{w}^{(k-1)}\right] \in \mathbb{R}^{m \times n}$ and consider the block sizes $(2,1, \ldots, 1)$. We claim that $A$ satisfies the nonnegative block-linear NSP of order $s^{*}$. Namely, assume that there exists a nonzero vector $v=\left(v_{1}, \ldots, v_{n}\right)^{\top} \in \mathcal{N}(A) \backslash\{0\}$ and $S \subseteq[k]$ with $|S| \leq s^{*}$ and $v_{\bar{S}} \leq 0$ such that $\sum_{i \in S} \mathbb{1}^{\top} v[i] \geq\left\|v_{\bar{S}}\right\|_{1,1}$. Since $v \in \mathcal{N}(A)$ and since the penultimate and the last row of $A$ only have a single nonzero entry, we have $v_{1}=v_{2}=0$. Hence, $\tilde{v}:=\left(v_{1}, v_{3}, \ldots, v_{n}\right)^{\top}$ is a nonzero vector in the null space of $A^{\diamond}=\left[w^{\prime}, \hat{w}^{(1)}, \ldots, \hat{w}^{(k-1)}\right]$ and violates the nonnegative linear NSP of order $s^{*}$ for $A^{\diamond}$. However, since the polytope $P$ and thus also the polytope $\operatorname{conv}\left\{w^{\prime}, \hat{w}^{(1)}, \ldots, \hat{w}^{(k-1)}\right\}$ are $\lfloor m / 2-1\rfloor$-neighborly (due to the pyramidal construction with respect to the apex $w^{\prime}$ ), this is a contradiction.

The nonnegative linear NSP of order $s^{*}$ does not hold for $A$ if $m \geq 12$, because the polytope $P^{\prime}:=\operatorname{conv}\left\{w^{\prime}, w^{\prime \prime}, \hat{w}^{(1)}, \ldots, \hat{w}^{(k-1)}\right\}$ is not $s^{*}$-neighborly. To see this, observe that any choice of vertices which includes $w^{\prime}$ and $w^{\prime \prime}$ cannot span a face, hence $P^{\prime}$ is not 2-neighborly, and this implies that $P^{\prime}$ is not $\lfloor m / 2-1\rfloor$-neighborly because of $m \geq 6$.

It remains to show that the unrestricted block-linear $\mathrm{NSP}_{q, 1}$ of order $s^{*}$ is not satisfied for $m \geq 12$. Assume that it is satisfied. Then for any $v=\left(v_{1}, \ldots, v_{n}\right)^{\top} \in \mathcal{N}(A) \backslash\{0\}$ and $S \subseteq[k]$ with $|S| \leq s^{*}$, we have $\|v[S]\|_{q, 1}<\|v[\bar{S}]\|_{q, 1}$ Restricting to $v_{1}=0$, the induced NSP-formula of order $s^{*}$ must also hold for any corresponding $\left(v_{2}, \ldots, v_{n}\right)^{\top} \in \mathcal{N}(\tilde{A})$, where $\tilde{A}$ results from $A$ by deleting the first column, i.e., $\tilde{A}=\left[w^{\prime \prime}, w^{(1)} \ldots, w^{(k-1)}\right]$. But this is a contradiction to the results of McMullen and Shephard from Remark 4.7, because we have $m \geq 12$ and thus $s^{*}=\lfloor m / 2-1\rfloor>\lfloor(m+1) / 3\rfloor$.

Remark 4.8. The construction in the proof can be generalized, for example to block sizes $\left(n_{1}, \ldots, n_{k}\right)=(\underbrace{2, \ldots, 2}_{r}, \underbrace{1, \ldots, 1}_{n-r})$ for fixed $r$ and sufficiently large $k$.

\section{Conclusion And Open Questions}

We have presented and discussed a comprehensive framework for recovery problems, which, in particular, allows to capture nonnegativity and positive semidefiniteness constraints. Building upon this framework, we have established generalized null space conditions, which has also allowed us to derive a systematic classification of broad classes of NSPs from a single generalization.

We close the paper by mentioning some open questions. An important aspect not covered in the present paper concerns the block-structure for vectors. Instead of exploiting the knowledge about the block-structure, it is possible to directly apply the methods and optimization problems for recovery of non-block-structured vectors, by disregarding any information about blocks. It is clear that in the setting of block-sparse vectors consisting 
of $k$ blocks, every block-s-sparse vector $x$ is also $\tilde{s}$-sparse in the classical sense, where $\tilde{s}$ is the sum of the $s$ largest block sizes of the $k$ blocks, since $x$ has at most $\tilde{s}$ nonzero elements. However, not every sparse vector is also block-sparse with respect to some block-structure. Thus, the conditions for uniform recovery of non-block-sparse vectors may be too strong for uniform recovery of all block-sparse vectors. For a short discussion in terms of the restricted isometry constant and property, and an illustrative example, see [15].

In the block-structured settings, an inner $\ell_{2}$-norm or an inner Frobenius norm is typically used in the recovery problems due to their robustness. Without using nonnegativity or positive semidefiniteness, the respective null space properties can be applied, since these hold for arbitrary inner norms, see Remark 3.8 and Corollary 4.2. If nonnegativity or positive semidefiniteness is exploited in the recovery problem, things seem to be different. In these cases, the null space properties only hold if the inner norm is given by the $\ell_{1}$-norm in the case of vectors or the nuclear norm in the case of matrices, see Theorem 3.4 and Corollary 4.1. Thus, an interesting line of future research would be to analyze what happens if another (inner) norm is used in these block-structured settings.

For non-block settings, however, using different norms often has side-effects. For example, in the classical case of sparse recovery, it is well-known that recovery using the $\ell_{q}$-norm and the optimization problem

$$
\min \left\{\|x\|_{q}: A x=b\right\}
$$

with $q>1$ already fails for 1 -sparse vectors, in general, whereas for $0<q<1$ the $\ell_{q^{-}}$ norm leads to favorable recovery properties [34], but results in an $\mathcal{N} \mathcal{P}$-hard optimization problem [20]. Note that the null space property (NSP) also characterizes uniform recovery using (5.1) when replacing the $\ell_{1}$-norm by the $\ell_{q}$-norm with $0<q<1$, see [18].

\section{ACKNOWLEDGEMENT}

We thank the anonymous reviewers for valuable comments and suggestions that helped to improve the style and presentation of the paper.

\section{REFERENCES}

[1] A. A. Ahmadi and G. Hall. DC decomposition of nonconvex polynomials with algebraic techniques. Math. Program., 169(1):69-94, 2018.

[2] J.-F. Cai and W. Xu. Guarantees of total variation minimization for signal recovery. Inf. Inference, $4(4): 328-353,2015$.

[3] E. J. Candès and D. L. Donoho. New tight frames of curvelets and optimal representations of objects with piecewise $C^{2}$ singularities. Commun. Pure Appl. Math., 57(2):219-266, 2004.

[4] E. J. Candès, Y. C. Eldar, D. Needell, and P. Randall. Compressed sensing with coherent and redundant dictionaries. Appl. Comput. Harmon. Anal., 31(1):59-73, 2011.

[5] T. F. Chan and J. J. Shen. Image processing and analysis: variational, PDE, wavelet, and stochastic methods, volume 94. SIAM, 2005.

[6] S. S. Chen, D. L. Donoho, and M. A. Saunders. Atomic decomposition by basis pursuit. SIAM J. Sci. Comput., 20(1):33-61, 1999.

[7] J. W. Chinneck. Feasibility and Infeasibility in Optimization: Algorithms and Computational Methods, volume 118 of Internat. Series in Oper. Research and Management Sciences. Springer, 2008.

[8] A. Cohen, W. Dahmen, and R. DeVore. Compressed sensing and best $k$-term approximation. J. Amer. Math. Soc., 22(1):211-231, 2009. 
[9] D. L. Donoho. Neighborly polytopes and sparse solutions of underdetermined linear equations. Technical Report 2005-4, Dept. of Statistics, Stanford Univ., 2005.

[10] D. L. Donoho and X. Huo. Uncertainty principles and ideal atomic decomposition. IEEE Trans. Inf. Theory, 47(7):2845-2862, 2001.

[11] D. L. Donoho and J. Tanner. Sparse nonnegative solution of underdetermined linear equations by linear programming. Proc. National Acad. Sci., 102(27):9446-9451, 2005.

[12] D. L. Donoho and J. Tanner. Sparse nonnegative solution of underdetermined linear equations by linear programming. Technical Report 2005-6, Dept. of Statistics, Stanford Univ., 2005.

[13] M. Elad, P. Milanfar, and R. Rubinstein. Analysis versus synthesis in signal priors. Inverse Probl., 23(3):947-968, 2007.

[14] Y. C. Eldar, P. Kuppinger, and H. Bölcskei. Block-sparse signals: Uncertainty relations and efficient recovery. IEEE Trans. Signal Process., 58(6):3042-3054, 2010.

[15] Y. C. Eldar and M. Mishali. Robust recovery of signals from a structured union of subspaces. IEEE Trans. Inf. Theory, 55(11):5302-5316, 2009.

[16] E. Elhamifar and R. Vidal. Block-sparse recovery via convex optimization. IEEE Trans. Signal Process., 60(8):4094-4107, 2012.

[17] M. Fazel. Matrix Rank Minimization with Applications. PhD thesis, Stanford University, 2002.

[18] S. Foucart and H. Rauhut. A Mathematical Introduction to Compressive Sensing. Applied and Numerical Harmonic Analysis. Birkhäuser/Springer, New York, 2013.

[19] M. Fukuda, M. Kojima, K. Murota, and K. Nakata. Exploiting sparsity in semidefinite programming via matrix completion I: General framework. SIAM J. Optim., 11(3):647-674, 2001.

[20] D. Ge, X. Jiang, and Y. Ye. A note on the complexity of $l_{p}$ minimization. Math. Program., 129(2):285$299,2011$.

[21] J. Gleeson and J. Ryan. Identifying minimally infeasible subsystems of inequalities. ORSA J. Comput., 2(1):61-63, 1990.

[22] R. Gribonval and M. Nielsen. Sparse representations in unions of bases. IEEE Trans. Inf. Theor., 49(12):3320-3325, 2003.

[23] K. Gröchenig. Foundations of time-frequency analysis. Applied and Numerical Harmonic Analysis. Birkhäuser, Boston, 2001.

[24] A. Juditsky, F. K. Karzan, and A. Nemirovski. On a unified view of nullspace-type conditions for recoveries associated with general sparsity structures. Linear Algebra Appl., 441:124-151, 2014.

[25] M. Kabanava and H. Rauhut. Cosparsity in compressed sensing. In H. Boche, R. Calderbank, G. Kutyniok, and J. Vybíral, editors, Compressed Sensing and its Applications: MATHEON Workshop 2013, pages 315-339. Springer, 2015.

[26] S. Keiper, G. Kutyniok, D. G. Lee, and G. E. Pfander. Compressed sensing for finite-valued signals. Linear Algebra Appl., 532:570-613, 2017.

[27] K. Kellner, M. E. Pfetsch, and T. Theobald. Irreducible infeasible subsystems of semidefinite systems. J. Optim. Theory Appl., 181(3):727-742, 2019.

[28] M. A. Khajehnejad, A. G. Dimakis, W. Xu, and B. Hassibi. Sparse recovery of nonnegative signals with minimal expansion. IEEE Trans. Signal Process., 59(1):196-208, 2011.

[29] L. Kong, J. Sun, and N. Xiu. S-semigoodness for low-rank semidefinite matrix recovery. Pacific J. Optim., 10(1):73-83, 2014.

[30] J.-H. Lange, M. E. Pfetsch, B. M. Seib, and A. M. Tillmann. Sparse recovery with integrality constraints. Discrete Applied Math., in press, DOI 10.1016/j.dam.2020.01.021, 2020.

[31] J. H. Lin and S. Li. Block sparse recovery via mixed $l_{2} / l_{1}$ minimization. Acta Math. Sin., 29(7):1401$1412,2013$.

[32] S. Mallat. A Wavelet Tour of Signal Processing, Third Edition: The Sparse Way. Academic Press, 3rd edition, 2008.

[33] P. McMullen and G. C. Shephard. Diagrams for centrally symmetric polytopes. Mathematika, 15(2):123-138, 1968. 
[34] N. Mourad and P. Reilly. Minimizing nonconvex functions for sparse vector reconstruction. IEEE Trans. Signal Process., 58(7):3485-3496, 2010.

[35] K. Nakata, K. Fujisawa, M. Fukuda, M. Kojima, and K. Murota. Exploiting sparsity in semidefinite programming via matrix completion II: implementation and numerical results. Math. Program., 95(2):303-327, Feb 2003.

[36] S. Nam, M. Davies, M. Elad, and R. Gribonval. The cosparse analysis model and algorithms. Appl. Comput. Harmon. Anal., 34(1):30-56, 2013.

[37] D. Needell and R. Ward. Stable image reconstruction using total variation minimization. SIAM J. Imaging Sci., 6(2):1035-1058, 2013.

[38] S. Oymak and B. Hassibi. New null space results and recovery thresholds for matrix rank minimization. In Proc. ISIT 2011, Preprint arXiv:1011.6326, 2010.

[39] B. Recht, M. Fazel, and P. Parrilo. Guaranteed minimum-rank solutions of linear matrix equations via nuclear norm minimization. SIAM Rev., 52(3):471-501, 2010.

[40] B. Recht, W. Xu, and B. Hassibi. Necessary and sufficient conditions for success of the nuclear norm heuristic for rank minimization. In 47th IEEE Conf. Decision and Control, pages 3065-3070, 2008.

[41] A. Ron and Z. Shen. Affine systems in $l_{2}\left(\mathbb{R}^{d}\right)$ : The analysis of the analysis operator. J. Funct. Anal., 148(2):408-447, 1997.

[42] I. W. Selesnick and M. A. Figueiredo. Signal restoration with overcomplete wavelet transforms: Comparison of analysis and synthesis priors. In Wavelets XIII, volume 7446, page 74460D. Internat. Society for Optics and Photonics, 2009.

[43] M. Stojnic. Compressed sensing of block-sparse positive vectors. Preprint, arXiv:1306.3977, 2013.

[44] M. Stojnic, F. Parvaresh, and B. Hassibi. On the reconstruction of block-sparse signals with an optimal number of measurements. IEEE Trans. Signal Process., 57(8):3075-3085, 2009.

[45] A. M. Tillmann and M. E. Pfetsch. The computational complexity of the restricted isometry property, the nullspace property, and related concepts in compressed sensing. IEEE Trans. Inf. Theory, 60(2):1248-1259, 2014.

[46] L. Vandenberghe and M. S. Andersen. Chordal graphs and semidefinite optimization. Foundations and Trends in Optimization, 1(4):241-433, 2015.

[47] Y. Zhang. A simple proof for recoverability of $\ell_{1}$-minimization (II): the nonnegativity case. Technical report TR05-10, Dept. of Computational and Applied Mathematics, Rice University, 2005.

[48] G. Ziegler. Lectures on Polytopes. Graduate Texts in Mathematics. Springer, New York, 1995.

(Janin Heuer) Technische Universität Braunschweig, Institut fÜr Analysis und Algebra, AG Algebra, Universitätsplatz 2, 38106 Braunschweig, Germany

E-mail address: janin.heuer@tu-braunschweig.de

(Frederic Matter*) Department of Mathematics, TU Darmstadt, Dolivostr. 15, 64293 DarmSTADT, GERMANY

E-mail address: matter@mathematik.tu-darmstadt.de

(Marc E. Pfetsch) Department of Mathematics, TU Darmstadt, Dolivostr. 15, 64293 DarmSTADT, Germany

E-mail address: pfetsch@mathematik.tu-darmstadt.de

(Thorsten Theobald) Goethe-Universität, FB 12 - Institut fÜr Mathematik, Postfach 11 19 32, 60054 Frankfurt am Main, Germany

E-mail address: theobald@math.uni-frankfurt.de

\footnotetext{
${ }^{*}$ Corresponding author.
} 